\title{
上地幔中的岩浆混合作用: 东太平洋海隆 $13^{\circ} \mathrm{N}$ 附近 玄武岩高 Mg\#橄榄石内熔体包裹体证据
}

张国良 ${ }^{\left(12^{*}\right.}$ ，江少卿 ${ }^{(3)}$ ，欧阳荷根 ${ }^{(12)}$ ，陈代庚 ${ }^{(4)}$ ，殷学博 ${ }^{(1)}$ ，王晓媛 ${ }^{(1)}$ ，汪小妹 ${ }^{(12)}$ ，曾志刚 ${ }^{\left(\mathbb{1}^{*}\right.}$

(1) 中国科学院海洋研究所海洋地质与环境重点实验室, 青岛 266071;

(2) 中国科学院研究生院, 北京 100049 ;

(3) 中国地质科学院地质研究所, 北京 100037 ;

(4) 有色金属华东地质勘查局, 南京 210007

*联系人, E-mail: tswc_zgl@163.com,zgzeng@ms.qdio.ac.cn

2009-08-24 收稿, 2009-12-01 接受

国家自然科学基金(批准号: 40830849)和国家大洋重大专项课题(编号: DYXM-115-02-1-03)资助项目

摘要 洋中脊玄武质岩浆中早期结晶产物：高 $\mathrm{Mg}$ \#橄榄石常捕获有早期熔体, 从而形成熔体包 裹体. 这些熔体包裹体记录了岩浆早期演化的重要信息. 本研究获得的东太平洋海隆(EPR) $13^{\circ} \mathrm{N}$ 附近 5 个站位玄武岩具有很小的 K/Ti (0.07 0.12), Tb/Lu (1.72 1.84), $\mathrm{Sm} / \mathrm{Nd}(0.310 \sim 0.332)$ 变化范围和相似的稀土元素配分型式, 说明亏损上地幔源区具有相似的矿物组成. 结合前人的 $\mathrm{Sr}$ 和 $\mathrm{Nd}$ 同位素分析结果表明这些玄武岩未经受明显的洋壳混染作用. 通过对校正檄览石分异 和“铁丢失”作用后获得的 65 个初始熔体包裹体组成分析, 表明这些熔体包裹体具有较寄主玄 武岩高的 $\mathrm{MgO}$ 含量, 因此, 可能是寄主岩浆早期不同演化阶段的产物, 从而能够指示岩浆较早 期阶段的演化过程. 寄主玄武岩尚处于橄榄石 + 斜长石共结结晶阶段, 但熔体包裹体具有较寄 主玄武岩更高的 $\mathrm{CaO} / \mathrm{Al}_{2} \mathrm{O}_{3}$ 比值, 且该比值与 $\mathrm{MgO}$ 正相关和 $\mathrm{Na}_{2} \mathrm{O}$ 负相关的事实表明寄主玄武 岩经历了较高压下的单斜辉石结晶分异. 同时, 根据单斜辉石结晶和熔体包裹体液相线组成计 算其平均结晶压力为 $(0.83 \pm 0.25) \mathrm{GPa}$, 说明这些熔体包裹体平均捕获于洋底以下 $24 \mathrm{~km}$ 左右的 上地幔中. 熔体包裹体的 $\mathrm{Ca}_{8} / \mathrm{Al}_{8}, \mathrm{Na}_{8}$ 与 $\mathrm{Fe}_{8}$ 之间良好的负相关性, 及其明显高于寄主玄武岩的 $\mathrm{Ca}_{8} / \mathrm{Al}_{8}, \mathrm{Na}_{8}, \mathrm{Fe}_{8}$ 和 $\mathrm{K} / \mathrm{Ti}$ 变化范围, 显示这些熔体包裹体形成于不同熔融程度和深度的岩浆混合 作用. 另外, 根据寄主玄武岩和熔体包裹体 $\mathrm{Ca}_{8} / \mathrm{Al}_{8}, \mathrm{Na}_{8}, \mathrm{Fe}_{8}$ 和 $\mathrm{K} / \mathrm{Ti}$ 的变化范围和平均值, 这些 玄武质岩浆仍需要在洋壳中经历其他组成端元的岩浆混合作用. 由此可见, 洋中脊玄武岩斑晶 内熔体包裹体组成的多样性并不都反映岩浆在洋壳内的混合和结晶作用, 形成于上地幔压力下 的橄榄石中熔体包裹体不能直接与其他矿物斑晶中熔体包裹体共同用于指示洋壳内的岩浆过 程. 本次高 $\mathrm{Mg}$ \#橄榄石中熔体包裹体研究证明东太平洋海隆下部地幔中的确存在不同来源深度 和熔融程度的岩浆混合作用.

洋中脊下部上地幔熔融形成的原始玄武质岩浆 不能够直接喷出洋底, 而是在喷出之前经历了不同 程度的分异结晶和混合作用 ${ }^{[1 ~ 8]}$, 因此玄武岩地球化 学能够用于反映幔源岩浆的形成和演化过程 ${ }^{[9]}$. 至今,
关键词

橄览石

熔体包裹体 洋中脊玄武岩 岩浆混合 上地幔 东太平洋海隆 
和绝热上升的假设之上 ${ }^{[8,10 \sim 13]}$. 例如, 过去关于蛇绿 岩套结构组成的研究表明, 莫霍面附近的纯橄岩形 成于玄武质岩浆与浅部地幔岩的相互作用, 这被认 为是岩浆绝热上升过程的表现 ${ }^{[14,15]}$; 地幔柱岩浆作 用模型研究表明，地幔熔融和岩浆上升过程大致符 合绝热过程 ${ }^{[16,17]}$; 过去在洋中脊获得的大量地幔岩 捕掳体被认为是地幔熔融残留相的代表, 而一般这 些残留相内不存在玄武质岩浆结晶的产物, 表明玄 武质岩浆在通过上地幔过程中未经历明显的分异结 晶作用 ${ }^{[18]}$. 岩浆绝热上升意味着岩浆实际温度高于 岩浆液相线(结晶)温度, 而地幔热导降温未能使岩浆 温度降至其液相线之下, 因此理想的绝热岩浆过程 不能分异出任何晶体. 一般认为只有岩浆侵人洋中 脊地壳中之后, 由于强烈的地壳热导降温作用才开 始大量结晶. 以往研究中, 玄武岩矿物斑晶内的熔体 包裹体常被用于指示岩浆在各演化阶段中的不同端 元，并反映岩浆在岩浆房内的混合-结晶作用 ${ }^{[19 ~ 23]}$. 可见, 在绝热岩浆过程这一理论框架下, 不存在岩浆 在地幔中演化过程的直接熔体包裹体记录. 目前对 岩浆在侵人洋壳之前上地幔中的演化过程了解很少.

但近年来，地质工作者开始认识到洋中脊玄武 质岩浆在侵人洋壳之前并不是理想的绝热过程, 而 是在通过上地幔的过程中经历了一定程度的降温和 分异结晶作用. 如, $\mathrm{Niu}$ 等人 ${ }^{[18]}$ 指出东太平洋海隆的 深海橄榄岩组成并不完全代表地幔熔融的残留相, 其中一部分高 $\mathrm{Mg}$ \#橄榄石形成于岩浆在通过上地幔 过程中的结晶作用; Eason 和 Sinton ${ }^{[24]}$ 认识到加拉帕 格斯(Galapágos) 扩张中心的高铝玄武岩是岩浆在地 幔中较高压力下结晶的产物; Falloon 等人 ${ }^{[25]}$ 通过橄 榄石温度计计算的原始玄武质岩浆 $(\mathrm{Mg} \approx 16 \%$ ，质量 分数, 下同)在 $1345^{\circ} \mathrm{C}$ 之下可以结晶出橄榄石, 而这 一温度高于一般正常洋中脊最上部地幔的温度, 使 得玄武质岩浆温度在地幔中就开始降至液相线之下， 因此必然会导致橄榄石的结晶. 另外, 相关研究指出 东太平洋海隆高 $\mathrm{Mg}$ \#橄榄石中的熔体包裹体常比与 之共生的斜长石 (低压结晶产物)熔体包裹体更原 始 ${ }^{[26]}$, 说明部分橄榄石熔体包裹体可能形成于更早 的岩浆演化阶段. 因此, 洋中脊玄武岩中的橄榄石斑 晶可能并不都形成于地壳中，其中一些(尤其是高 $\mathrm{Mg}$ \#橄榄石)可能形成于岩浆在上地幔中的高压分异 结晶作用. 因为原始玄武质岩浆可结晶出较高 $\mathrm{Mg} \#$ 的橄榄石, 而随着分异结晶进行, 晶出的橄榄石 $\mathrm{Mg} \#$
逐渐降低. 因此, 较高 $\mathrm{Mg}$ \#的橄榄石最有可能来自于 较早期(相对于岩浆在洋壳内结晶而言)岩浆在地幔 中结晶的产物. 可见, 尽管高 $\mathrm{Mg} \#$ 橄榄石内的熔体 包裹体一般可反映地壳内的岩浆演化过程, 但其中 一些也可能反映了岩浆在上地幔而不是地壳中的演 化过程. 本研究获得了来自于东太平洋海隆 $13^{\circ} \mathrm{N}$ 附 近 5 个站位的玄武岩, 分析了其中高 $\mathrm{Mg}$ \#橄榄石内熔 体包裹体组成，并试图通过这些熔体包裹体获得玄 武质岩浆在地幔中分异结晶的直接证据, 并研究可 能发生在地幔中的岩浆演化过程.

\section{1 地质背景}

研究区位于 Orozco 断裂带和另一个小断裂带 $\left(11^{\circ} 49^{\prime} \mathrm{N}\right)$ 之间(图 1), 属快速扩张洋中脊, 扩张速率为 10 11 cm/a, 海隆两侧半扩张速率不对称(西侧 6.5 $\mathrm{cm} / \mathrm{a}$, 东侧 $4.5 \mathrm{~cm} / \mathrm{a})^{[27]}$. Choukroune 等人 ${ }^{[27]}$ 根据构造 特征和基底地形将本研究区分为 4 个部分：(1) 位于中 部地堑的活动型火山带; (2) 构成中央地堑并向隆起 两侧延伸 $2 \mathrm{~km}$ 的活动构造带; (3) 距海隆 $2 \mathrm{~km}$ 以外 由不连续的地垒和地堑构成的非活动构造带; (4) 距 离海隆轴部两侧各 $20 \mathrm{~km}$ 以内的离轴火山(海山). 研 究区北端存在一个小型海山(直径 $750 \mathrm{~m}$, 高度 $80 \mathrm{~m}$ ). 洋中脊轴呈轴向地堑结构产出, 地堑宽度约 200 $600 \mathrm{~m}$, 深度 $20 \sim 50 \mathrm{~m}$, 地堑底部平均水深 $2630 \mathrm{~m}$, 其中分布有许多裂隙, 海隆内部主要由玄武岩组成, 沉积物覆盖很少 ${ }^{[28,29]}$. 该区洋壳下 1 2 km 处存在透 镜状的岩浆房, 其沿洋中脊轴部方向断续分布, 覆盖 于厚且宽的碎晶带之上 ${ }^{[4]}$.

\section{2 样品和测试方法}

玄武岩样品采自 EPR $12^{\circ} 44^{\prime} \mathrm{N} \sim 12^{\circ} 51^{\prime} \mathrm{N}$ 间的轴部 地堑, 拖网站位从南向北分别为 E42, E20, E15, E11 和 E13. 由薄片分析可知所有样品中均含有极少量橄 榄石和斜长石斑晶, 两者分别约占斑晶总量 10\% 20\%和 80\% 90\%. 橄榄石斑晶根据其粒径，可 分为 $<1 \mathrm{~mm}$ 和 $1 \sim 2 \mathrm{~mm}$ 两个粒级, 一个薄片内粒径 $1 \sim 2 \mathrm{~mm}$ 的橄榄石斑晶一般数量较少并部分含有熔体 包裹体; 而 $<1 \mathrm{~mm}$ 的斑晶占绝大多数(橄榄石斑晶颗 粒数 $90 \%$ 以上), 且几乎均为橄榄石微晶. 这两个粒 级的橄榄石斑晶明显结晶于两个不同的阶段. 所有 玄武岩基质中还有大量橄榄石、斜长石和单斜辉石微 晶, 但是它们未出现于玄武岩玻璃中, 表明基质中的 


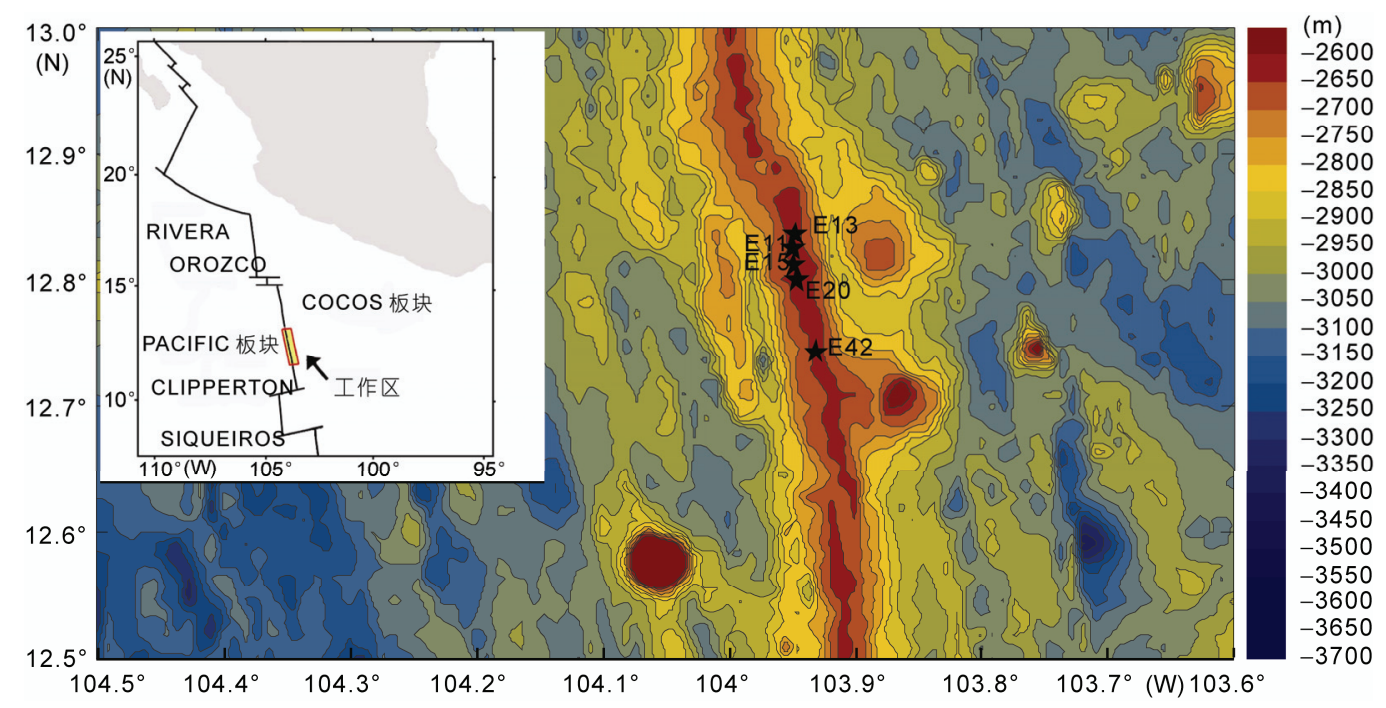

图 1 东太平洋海隆 $13^{\circ} \mathrm{N}$ 附近等深线和采样站位图

等深线数据来自 http://www.geomapapp.org

微晶是由于岩浆遇海水冷凝时快速成核而成，而玄 武岩玻璃质由于瞬间内冷凝而未能结晶任何微晶. 橄榄石斑晶内常存在一个或数个熔体包裹体, 多呈 不规则球形, 直径多介于几到几十微米, 且其中未出 现结晶化现象(图 2).

本研究是在薄片上并通过电子探针分析熔体包 裹体成分. 包裹体选择是通过透射-反射一体偏光显 微镜, 先通过透射偏光找到包裹体, 再在反射镜下检 验包裹体是否出露于探针片表面, 然后分析出露于 探针片表面的包裹体. 为了分析保存完好并具代表
性的早期橄榄石结晶形成的熔体包裹体, 我们进行 了以下选择: 个别橄榄石因为存在裂隙, 其中熔体包 裹体可能并不完全封闭，应予排除; 另外，只选择粒 径 $>1 \mathrm{~mm}$ 的橄榄石斑晶和其中熔体包裹体在背散射 电子图像中成分均一的熔体包裹体进行分析. 在满 足以上条件下, 本研究分析了 65 个橄榄石斑晶内的 熔体包裹体. 主矿物橄榄石和熔体包裹体电子探针 分析是在中国地质科学院矿产资源研究所通过 JXA8800R (日本 JEOL 公司) 电子探针仪进行. 工作 条件为: 高压 $20 \mathrm{keV}$ ，电子束束流 $2 \times 10^{-8} \mathrm{~A}$. 对于主矿
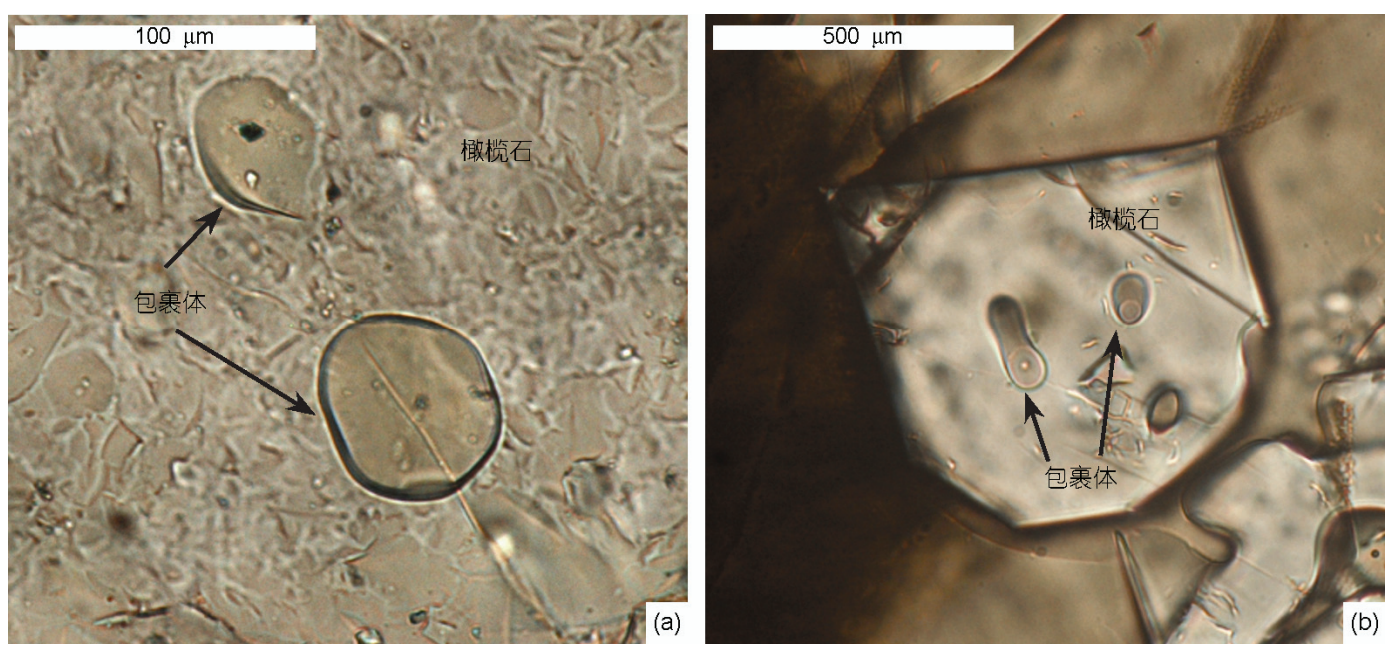

图 2 代表性橄榄石熔体包裹体单偏光显微照片

(a)和(b)分别为玄武岩 E15 和 E13 橄榄石内的熔体包裹体. 显然, 熔体包裏体内未出现结晶化现象 
物橄榄石和熔体包裹体中主量元素(含量>1\%)电子探 针分析时间约为 $30 \mathrm{~s}$, 而对于次量元素, 如 $\mathrm{SO}_{3}$, $\mathrm{MnO}_{2}, \mathrm{~K}_{2} \mathrm{O}$ 和 $\mathrm{Cr}_{2} \mathrm{O}_{3}$, 分析时间约为 $60 \mathrm{~s}$, 以获得所 有元素较高的分析精度. 标准样品采用国家电子探 针标准样品: 橄榄石 (GSB 01-1415-2001)、辉石 $(\mathrm{K} 17)$ 、钻长石 $(\mathrm{K} 3)$ 、玄武玻璃 $(\mathrm{K} 71)$. 修正方法采用 ZAF 法. 电子探针对造岩矿物组成元素的分析检 测限为 $0.01 \%$, 测试过程通过标准样品检测仪器稳 定性, 可获得含量 $1.0 \%$ 以上的主量元素重复性好于 $\pm 1 \%$. 因为这 5 个站位玄武岩全岩组成已进行过报道, 常量、微量元素和稀土元素数据选自 Zhang 等人 ${ }^{[30]}$ (表 1).

\section{3 结果}

由表 2 可知, 这些橄榄石中包裹体具有很低的 $\mathrm{MgO}$ 含量 $(2.05 \%$ 7.69\%)、相对寄主玄武岩全岩较高的 $\mathrm{CaO}(12.3 \% \sim 16.2 \%), \mathrm{Al}_{2} \mathrm{O}_{3}(14.3 \% \sim 19.3 \%), \mathrm{TiO}_{2}(1.33 \%$ $2.82 \%)$ 等的含量和较高的 $\mathrm{CaO} / \mathrm{Al}_{2} \mathrm{O}_{3}$ 比值 $(0.60 \sim 1.04)$. 这些熔体包裏体中具强挥发性的 $\mathrm{S}\left(\mathrm{SO}_{3}\right.$ 介于 $0.22 \%$ 0.72\%)含量远高于一般所报道的洋中脊玄武 岩 $\left(\mathrm{SO}_{3}<0.2 \%\right)^{[31 \sim 33]}$, 说明初始形成的包裹体被主矿 物橄榄石完整地保存, 而未受岩浆演化和喷发过程 中脱气作用影响.

我们将 $\mathrm{FeO}_{\mathrm{t}}$ 和 $\mathrm{CaO}$ 与 $\mathrm{MgO}$ 作相关性图解(图 3).

表 1 玄武岩全岩主量 $(\%)$ 、稀土元素组成 $(\mu \mathrm{g} / \mathrm{g})$ 和微量元素比值

\begin{tabular}{|c|c|c|c|c|c|}
\hline 样品 & E15 & E13 & E11 & E20 & $\mathrm{E} 42$ \\
\hline $\mathrm{SiO}_{2}$ & 49.7 & 50.2 & 50.9 & 50.6 & 51.4 \\
\hline $\mathrm{TiO}_{2}$ & 1.18 & 1.47 & 1.52 & 1.45 & 1.61 \\
\hline $\mathrm{Al}_{2} \mathrm{O}_{3}$ & 16.9 & 15.6 & 14.5 & 15.8 & 14.8 \\
\hline $\mathrm{FeO}_{\mathrm{t}}$ & 8.29 & 9.36 & 10.8 & 9.29 & 10.0 \\
\hline $\mathrm{MnO}$ & 0.15 & 0.17 & 0.14 & 0.17 & 0.18 \\
\hline $\mathrm{MgO}$ & 9.15 & 8.25 & 7.42 & 7.86 & 7.37 \\
\hline $\mathrm{CaO}$ & 11.8 & 11.7 & 11.6 & 11.7 & 11.4 \\
\hline $\mathrm{Na}_{2} \mathrm{O}$ & 2.61 & 2.85 & 2.82 & 2.80 & 2.90 \\
\hline $\mathrm{K}_{2} \mathrm{O}$ & 0.08 & 0.14 & 0.14 & 0.14 & 0.19 \\
\hline $\mathrm{P}_{2} \mathrm{O}_{5}$ & 0.11 & 0.15 & 0.14 & 0.15 & 0.18 \\
\hline 总量 & 99.97 & 99.89 & 99.98 & 99.96 & 100.03 \\
\hline $\mathrm{CaO} / \mathrm{Al}_{2} \mathrm{O}_{3}$ & 0.70 & 0.75 & 0.80 & 0.74 & 0.77 \\
\hline $\mathrm{K} / \mathrm{Ti}$ & 0.07 & 0.10 & 0.09 & 0.10 & 0.12 \\
\hline $\mathrm{Tb} / \mathrm{Lu}$ & 1.84 & 1.77 & 1.72 & 1.79 & 1.76 \\
\hline $\mathrm{Sm} / \mathrm{Nd}$ & 0.332 & 0.326 & 0.313 & 0.311 & 0.310 \\
\hline $\mathrm{Ca}_{8}$ & 11.3 & 11.6 & 12.0 & 11.8 & 11.8 \\
\hline $\mathrm{Al}_{8}$ & 16.0 & 15.4 & 15.1 & 15.9 & 15.3 \\
\hline $\mathrm{Na}_{8}$ & 2.81 & 2.89 & 2.71 & 2.77 & 2.78 \\
\hline $\mathrm{Fe}_{8}$ & 9.56 & 9.71 & 9.84 & 9.08 & 8.98 \\
\hline $\mathrm{Ca}_{8} / \mathrm{Al}_{8}$ & 0.71 & 0.75 & 0.80 & 0.74 & 0.77 \\
\hline $\mathrm{Fe}_{8}$ & 9.6 & 9.7 & 9.8 & 9.1 & 9.0 \\
\hline$F_{\text {melt }}$ & 15.7 & 15.9 & 17.5 & 16.3 & 16.8 \\
\hline $\mathrm{La}$ & 2.92 & 3.92 & 4.15 & 4.20 & 4.94 \\
\hline $\mathrm{Ce}$ & 9.50 & 12.2 & 12.1 & 11.8 & 15.5 \\
\hline $\operatorname{Pr}$ & 1.62 & 1.99 & 1.98 & 1.97 & 2.47 \\
\hline $\mathrm{Nd}$ & 8.61 & 10.7 & 11.0 & 10.2 & 13.2 \\
\hline $\mathrm{Sm}$ & 2.86 & 3.49 & 3.44 & 3.17 & 4.09 \\
\hline $\mathrm{Eu}$ & 1.09 & 1.25 & 1.30 & 1.24 & 1.44 \\
\hline $\mathrm{Gd}$ & 3.53 & 4.24 & 4.66 & 4.28 & 5.23 \\
\hline $\mathrm{Tb}$ & 0.70 & 0.85 & 0.86 & 0.77 & 1.02 \\
\hline Dy & 4.62 & 5.29 & 6.14 & 5.09 & 6.42 \\
\hline Ho & 0.96 & 1.16 & 1.27 & 1.12 & 1.38 \\
\hline $\mathrm{Er}$ & 2.77 & 3.29 & 3.36 & 3.08 & 3.93 \\
\hline $\mathrm{Tm}$ & 0.41 & 0.48 & 0.50 & 0.45 & 0.57 \\
\hline $\mathrm{Yb}$ & 2.54 & 3.13 & 3.39 & 2.98 & 3.65 \\
\hline $\mathrm{Lu}$ & 0.38 & 0.48 & 0.50 & 0.43 & 0.58 \\
\hline
\end{tabular}


随着橄榄石的结晶分离, 首先 $\mathrm{FeO}_{\mathrm{t}}$ 随着 $\mathrm{MgO}$ 降低 而升高, 当 $\mathrm{MgO}$ 低于 $5 \%$ 时则 $\mathrm{FeO}_{\mathrm{t}}$ 随着 $\mathrm{MgO}$ 降低 而降低(图 3(a)). 因为 $\mathrm{MgO}$ 具有相对 $\mathrm{FeO}$ 具有在橄 榄石与熔体之间较高的分配系数 $\left(\mathrm{Kd}^{\mathrm{Mg}-\mathrm{Fe}} \approx 3.0\right)^{[34]}$, 最初结晶的橄榄石为高 $\mathrm{MgO}(>45 \%)$ 和低 $\mathrm{FeO}(<10 \%)$, 因此可导致岩浆中 $\mathrm{MgO}$ 降低而 $\mathrm{FeO}$ 升高. 随着分异 结晶作用进行, 晶出橄榄石中 $\mathrm{MgO}$ 含量降低, 而 $\mathrm{FeO}$ 升高 (如橄榄石 $\mathrm{Mg}$ \#为 82 时, 其中 $\mathrm{FeO}$ 可达 $17 \%$ ). $\mathrm{CaO}$ 与 $\mathrm{MgO}$ 表现出明显的负相关(图 3(b)). 随着包裏体向周围晶出橄榄石 (其中 $\mathrm{MgO}$ 约 35\% 45\%), 其 $\mathrm{MgO}$ 含量大大降低, 如果同时有高 $\mathrm{CaO}$ 矿物结晶, 则必然有 $\mathrm{CaO}$ 随着 $\mathrm{MgO}$ 降低而降 低(正相关). 因为单斜辉石和基性斜长石中 $\mathrm{CaO}$ 均 约为 17\% 20\%, 如果单斜辉石和基性斜长石发生结 晶必然会形成 $\mathrm{MgO}-\mathrm{CaO}$ 正相关, 由此可见, 这些未 校正矿物分异结晶作用的包裹体 $\mathrm{MgO}-\mathrm{CaO}$ 负相关 性, 显示出只有橄榄石结晶, 而辉石和斜长石均未 参与分异结晶.

\section{4 讨论}

\section{1 源区特征和地売混染作用}

一般认为对于组成相对稳定的洋中脊地幔二辉 橄榄岩而言, 原始玄武质岩浆组成只受部分熔融条 件影响 ${ }^{[35,36]}$. 原始玄武质岩浆的 $\mathrm{Na}_{2} \mathrm{O}$ 和 $\mathrm{CaO} / \mathrm{Al}_{2} \mathrm{O}_{3}$ 只受熔融程度控制, 而不受熔融压力影响, 因此可用
以指示部分熔融程度大小. 但在实际应用中, 因为 $\mathrm{Na}_{2} \mathrm{O}, \mathrm{CaO}$ 和 $\mathrm{Al}_{2} \mathrm{O}_{3}$ 值都不同程度地受到岩浆分异结 晶作用影响，即玄武岩 $\mathrm{Na}_{2} \mathrm{O}, \mathrm{CaO}$ 和 $\mathrm{Al}_{2} \mathrm{O}_{3}$ 值并不代表 最初的原始岩浆组成; 为了使不同岩浆之间具有可 比性, 国际上通用的最好办法就是按照分异结晶趋 势将其校正至一固定的 $\mathrm{MgO}$ 处(一般选择 $\mathrm{MgO}=8 \%$ ), 校正后的 $\mathrm{Na}_{8}, \mathrm{Ca}_{8}$ 和 $\mathrm{Al}_{8}$ 就可以很好地指示部分熔融 程度 ${ }^{[35,37 ~ 40]}$. 因为原始玄武质岩浆的主量元素组成 变化只取决于二辉橄榄岩熔融物理化学条件 ${ }^{[30,37]}$, 二辉橄榄岩熔融产生的岩浆 $\mathrm{CaO}$ 和 $\mathrm{Al}_{2} \mathrm{O}_{3}$ 含量随着 熔融压力会发生同步变化, 但随着部分熔融程度增 大会分别升高和降低, 因此 $\mathrm{CaO} / \mathrm{Al}_{2} \mathrm{O}_{3}$ 比值不仅消除 了压力效应影响, 而且可以指示部分熔融程度大小. 另外, $\mathrm{Na}_{2} \mathrm{O}$ 受熔融程度影响很敏感, 呈现负相关关 系, 而几乎不受压力影响, 因此 $\mathrm{Na}_{2} \mathrm{O}$ 本身就能敏感 地指示部分熔融程度.

5 个站位样品均为拉斑玄武岩. 根据表 1 , 玄武岩 $\mathrm{MgO}$ 介于 $7.37 \%$ 9.15\%之间, 表明这些玄武岩经历 了不同程度的演化. 实验岩石学结论表明, 地幔橄榄 岩熔融形成原始洋中脊玄武质岩浆过程中, $\mathrm{K}$ 和 $\mathrm{Ti}$ 的 行为极为相似 ${ }^{[36,37]}$, 原始岩浆中 $\mathrm{K} / \mathrm{Ti}$ 比值在熔融过 程中不随熔融条件(部分熔融程度和压力)而变化, 且 在分异结晶时不受矿物分离结晶影响 ${ }^{[37]}$, 因此, K/Ti 可以理想地反映源区亏损程度. 稀土元素 $\mathrm{Tb}$ 与 $\mathrm{Lu}$ 和 $\mathrm{Sm}$ 与 $\mathrm{Nd}$ 具有相似的不相容性, 且在地幔熔融和岩浆
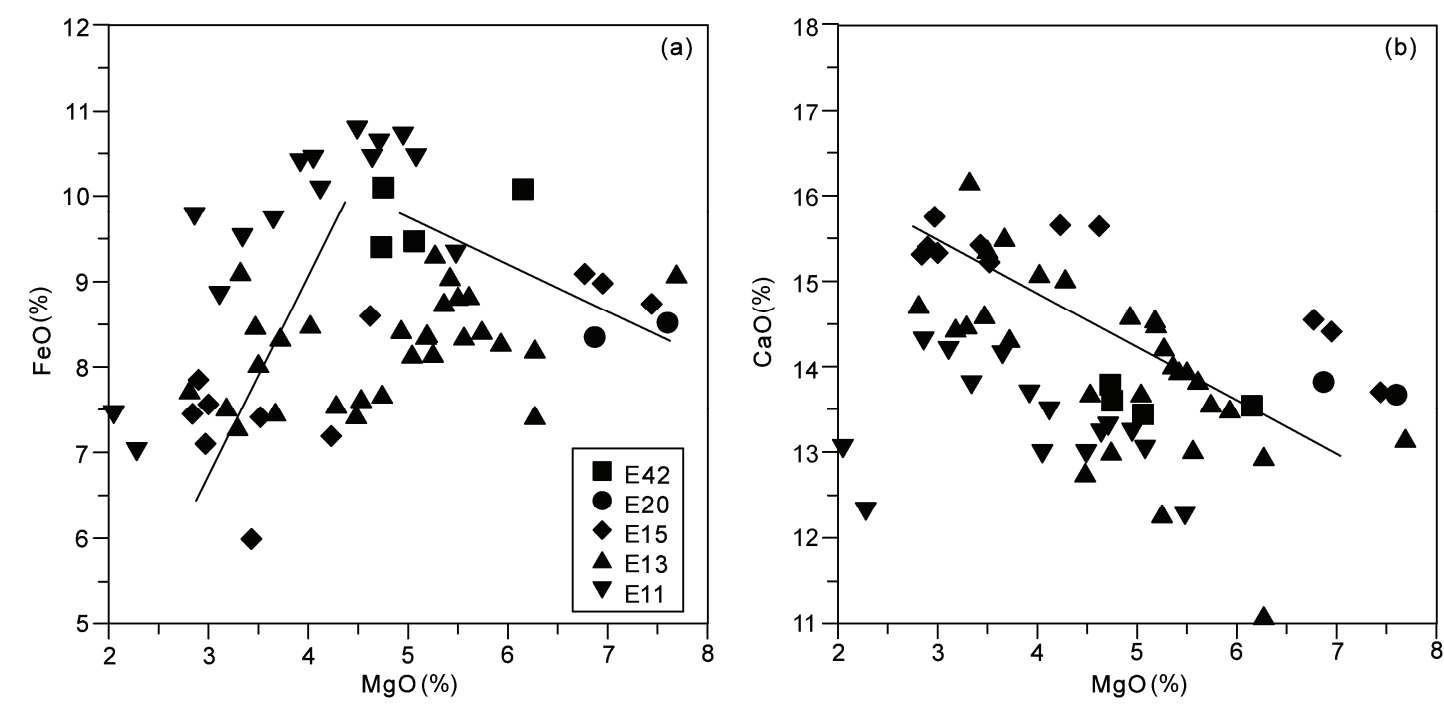

图 3 橄榄石熔体包裹体中(a) $\mathrm{FeO}_{\mathrm{t}}-\mathrm{MgO}$ 和 (b) $\mathrm{CaO}-\mathrm{MgO}$ 相关性变化图

$\mathrm{FeO}_{\mathrm{t}}$ 随着 $\mathrm{MgO}$ 先升高后降低的变化趋势和 $\mathrm{CaO}$ 作为不相容元素在熔体包裹体中富集, 均表明熔体包裹体被捕获后只结晶出了主矿物橄榄石 
结晶过程中化学行为极为相似, 因此 $\mathrm{Tb} / \mathrm{Lu}$ 和 $\mathrm{Sm} / \mathrm{Nd}$ 也可以反映源区组成特征. 由表 1 可知, 这些玄武岩 $\mathrm{K} / \mathrm{Ti}(0.07 \sim 0.12), \mathrm{Tb} / \mathrm{Lu}(1.72 \sim 1.84)$ 和 $\mathrm{Sm} / \mathrm{Nd}(0.310 \sim 0.332)$ 的变化范围都很小, 显示玄武岩上地幔源区亏损程 度和矿物组成一致.

研究区 (EPR $13^{\circ} \mathrm{N}$ 附近) $\mathrm{Sr}$ 和 $\mathrm{Nd}$ 同位素组成变化 范围几乎重叠, 并与其测试不确定度范围相当 $\left({ }^{87} \mathrm{Sr} /\right.$ ${ }^{86} \mathrm{Sr}$ 和 ${ }^{143} \mathrm{Nd} /{ }^{144} \mathrm{Nd}$ 比值变化 $\left.\mathrm{RSD}<10^{-4}\right)^{[41,42]}$. 根据 Macdougall 等人 ${ }^{[41]}$, Castillo 等人 ${ }^{[43]}$, Chaussidon 等人 ${ }^{[44]}$ 和 Hamelin 等人 ${ }^{[45]}$, 研究区玄武岩 ${ }^{87} \mathrm{Sr} /{ }^{86} \mathrm{Sr}$ 为 0.70251 $0.70262,{ }^{143} \mathrm{Nd} /{ }^{144} \mathrm{Nd}$ 为 $0.513107 \sim 0.513159$. 由于 $\mathrm{Sr}$ 和 $\mathrm{Nd}$ 同位素可以灵敏地指示洋中脊玄武岩受围岩混染 的程度 ${ }^{[46]}$, 可见本研究区玄武岩未受明显的混染和地 幔不一致性影响. 另外, 薄片分析未发现熔蚀的斑晶 矿物, 也表明岩浆未受围岩混染影响.

\section{2 初始包裹体还原}

获得橄榄石内初始熔体包裹体组成的方法(校正 主矿物后期结晶分异作用)一般有两种. 一种是, 在 显微镜下直接加热直至包裹体完全均一化, 这种方 法的最大困难在于包裹体均一温度的确定. 温度过 高会导致包裹体周围主矿物的回加过多, 温度过低 则又会导致其主矿物的回加不完全. 一般做法是将 包裹体中气泡恰好消失时的温度作为其初始捕获温 度 ${ }^{[47]}$. 但是, 洋中脊玄武岩中橄榄石熔体包裹体内 常无挥发份气泡, 如, 本研究中绝大多数橄榄石熔体 包裹体中不存在气泡; 另外, 正如 Kress 和 Ghiorso ${ }^{[48]}$ 所指出, 这个挥发分饱和的假设并不总是成立的, 包 裹体中挥发分丢失会导致加热温度过低.

洋中脊玄武岩是由岩浆喷出洋底并与海水接触 后迅速冷凝而成, 其中包裹体直接冷凝为玻璃质, 因 此成分均一. Ford 等人 ${ }^{[34]}$ 报道的用于计算岩浆液相 线橄榄石组成的 $\mathrm{Kd}^{\mathrm{Mg}-\mathrm{Fe}}$ 公式被公认为是检测橄榄石 是否与岩浆平衡的很好工具. 本研究据此计算了与 未回加橄榄石之前包裹体平衡的液相线橄榄石 $\mathrm{Mg} \#$ (网络版附表 1), 其明显低于实际获得的包裏体周围 主矿物橄榄石 $\mathrm{Mg}$, 表明主矿物橄榄石与包裹体之间 远未达平衡, 因此可知包裹体受后期扩散-重平衡作 用影响较小. 这种包裹体的初始成分还原可以通过第 二种方法: 热力学结晶程序计算. 此方法假设熔体包 裹体通过分离结晶的形式向包裹体壁结晶出主矿物. 热力学计算程序, 如 PETROLOG ${ }^{[49]}$ 和 FEO_EQ2.exe $\mathrm{e}^{[50]}$
等, 以集成的大量实验岩石学数据为基础, 可根据岩 浆化学组成计算液相线矿物组成. 本研究利用 FEO_EQ2.exe ${ }^{[50]}$ 计算初始熔体包裹体组成. 此方法通 过分步将橄榄石添加至包裹体内, 对于每个元素符合 以下等式:

\section{新熔体内质量分数 $(\%)=$ 原熔体内质量分数 $(\%) \times$}

$$
(1-X)+\text { 橄榄石内质量分数 }(\%) \times X \text {, }
$$

(1)式中 $X$ 为每步计算过程的分异结晶程度，一般选 $X=0.01 \%$. 新熔体内质量分数 $(\%)$ 是指利用 Ford 等 ${ }^{[34]}$ 添加 $0.01 \%$ 质量的平衡橄榄石后, 再根据(1)式计算 出的结果; 此后, 重新将获得的新熔体作为原熔体, 并继续添加 $0.01 \%$ 的平衡橄榄石. 本研究中选择包裹 体附近主矿物橄榄石的最高 $\mathrm{Mg}$ \# (网络版附表 2) 作为 停止计算条件. 实际计算过程中, 当计算获得的新熔 体液相线橄榄石 $\mathrm{Mg}$ \#与指定橄榄石的最高 $\mathrm{Mg} \#$ 值偏 差小于 $0.000001 \mathrm{~mol} \%$ (摩尔分数)时停止计算, 此时 的新熔体即为初始包裹体组成 (计算方法可参照 Zhang 等人 ${ }^{[51]}$ 和 Danyushevsky 等人 ${ }^{[50]}$ ).

使用 FEO_EQ2.exe 计算的具体方法和步骤如下: (1) 该计算需输人包裹体常量元素组成和主矿物 $\mathrm{Mg} \#$ 值, 并指定相应的初始 $\mathrm{FeO}_{\mathrm{t}}$ 值, 我们利用 Ford 等人 ${ }^{[34]}$ 报道的 $\mathrm{Mg}-\mathrm{Fe}$ 配分公式计算了主矿物橄榄石 与熔体包裹体平衡时的 $\mathrm{FeO}_{\mathrm{t}}$, 以作为其初始值; (2) 选择 Ford 公式和适合于洋中脊玄武质岩浆的 $\mathrm{Ca}, \mathrm{Mn}$ 和 $\mathrm{Cr}$ 校正公式; (3) 输人氧逸度控制的 $\mathrm{Fe}^{2+} / \mathrm{Fe}^{3+}$ 比值, 因为水分对岩浆系统的氧逸度影响很大 ${ }^{[52 ~ 54]}$, Danyushevsky 等人 ${ }^{[50]}$ 报道的 $\mathrm{Fe}^{2+} / \mathrm{Fe}^{3+}$ 只适用于控制水分 近饱和 $\left(\mathrm{H}_{2} \mathrm{O}\right.$ 约 2\% 6\%)的弧后盆地玄武质岩浆的氧逸 度, 而洋中脊玄武质岩浆被认为是无水系统(水含量 $<0.2 \%)$, 我们通过洋中脊玄武岩中常用的 $\mathrm{QFM}\left(\mathrm{SiO}_{2}\right.$ $\left.\mathrm{Fe}_{2} \mathrm{SiO}_{4}-\mathrm{Fe}_{3} \mathrm{O}_{4}\right)^{[55,56]}$ 氧逸度控制公式计算 $\mathrm{Fe}^{2+} / \mathrm{Fe}^{3+}$ 比 值; (4) 运行计算程序.

由表 3 可知，校正橄榄石分异结晶作用后的初始 包裹体多具有相对寄主玄武岩较高的 $\mathrm{MgO}$ 含量, 因 此代表了这些玄武岩的母岩浆, 并可以用于研究岩 浆的早期演化过程. 获得的初始熔体包裹体中 $\mathrm{SiO}_{2}$ 分别与 $\mathrm{CaO}, \mathrm{MgO}, \mathrm{Na}_{2} \mathrm{O}, \mathrm{TiO}_{2}, \mathrm{~K}_{2} \mathrm{O}$ 和 $\mathrm{SO}_{3}$ 表现出良 好的相关性(图 4). $\mathrm{SiO}_{2}$ 与其他元素的良好相关性表 明, 这些包裹体形成一个连续的演化系列, 证明此方 法有效地校正了熔体包裹体的后期橄榄石分异作用. $\mathrm{CaO}$ 随着分异结晶作用进行逐渐降低(图 4(a)), 说明 
有高 $\mathrm{Ca}$ 矿物 (斜长石和/或辉石)参与了结晶作用. $\mathrm{MgO}$ 与 $\mathrm{SiO}_{2}$ 之间的负相关性显示橄榄石曾参与结晶 作用(图 4(b)). $\mathrm{Na}_{2} \mathrm{O}, \mathrm{TiO}_{2}, \mathrm{~K}_{2} \mathrm{O}$ 和 $\mathrm{SO}_{3}$ 随着分异结晶 作用均连续变化, 并逐渐在岩浆中富集(图 4 (c) (f)).

\section{3 熔体包裹体结晶深度}

这些代表母岩浆组成的熔体包裹体具有相对寄 主玄武岩很高的 $\mathrm{CaO} / \mathrm{Al}_{2} \mathrm{O}_{3}$ 比值 $\left(\mathrm{CaO}\right.$ 和 $\mathrm{Al}_{2} \mathrm{O}_{3}$ 在橄榄 石中均为微量元素, 因此橄榄石结晶对 $\mathrm{CaO} / \mathrm{Al}_{2} \mathrm{O}_{3}$ 比 值影响很小), 说明这些寄主玄武岩一定经历了单斜 辉石分异作用. 如果只根据寄主玄武岩化学组成, 无 法确定其是否经历过单斜辉石结晶. 由于单斜辉石 结晶对压力的敏感性, 在岩浆上升到地表过程中, 随 着压力减小, 高压时产生的单斜辉石结晶作用在低 压下将受到抑制 ${ }^{[51]}$. 多数形成于深部的单斜辉石会 在岩浆过程中被重力作用分离出去, 但即使岩浆中
还有剩余的单斜辉石斑晶, 也会随着岩浆压力减小 趋于不稳定, 从而会被岩浆溶解 ${ }^{[51,57,58]}$.

另外, 包裹体中 $\mathrm{CaO} / \mathrm{Al}_{2} \mathrm{O}_{3}$ 比值与 $\mathrm{MgO}$ 和 $\mathrm{Na}_{2} \mathrm{O}$ 表现为正相关 (图 5), 这是因为随着分异结晶作用的 进行 ( $\mathrm{MgO}$ 降低, $\mathrm{Na}_{2} \mathrm{O}$ 升高), 单斜辉石分异结晶导致 了 $\mathrm{CaO} / \mathrm{Al}_{2} \mathrm{O}_{3}$ 比值的降低, 进一步说明图 4(a)指示的 高 $\mathrm{Ca}$ 结晶矿物是单斜辉石.

可见, 本研究 5 个站位玄武岩的母岩浆除在洋壳 内经历低压分异结晶之外, 至少还经历了一次深部 (地幔)橄榄石和辉石的分异结晶作用. 为了确定这些 熔体包裹体在地幔中被橄榄石捕获时的深度，我们 根据单斜辉石对结晶压力依赖性计算了岩浆结晶压 力. 我们通过 Yang 等人 ${ }^{[59]}$ 报道的常用于计算洋中脊 体系的压力计，以计算这些包裹体饱和单斜辉石时 的压力. 此方法被广泛认为可以准确反映低 $\mathrm{H}_{2} \mathrm{O}$ 拉 斑玄武质岩浆的结晶压力, 其计算误差 $1 \mathrm{hPa}^{[60 \sim 65]}$.
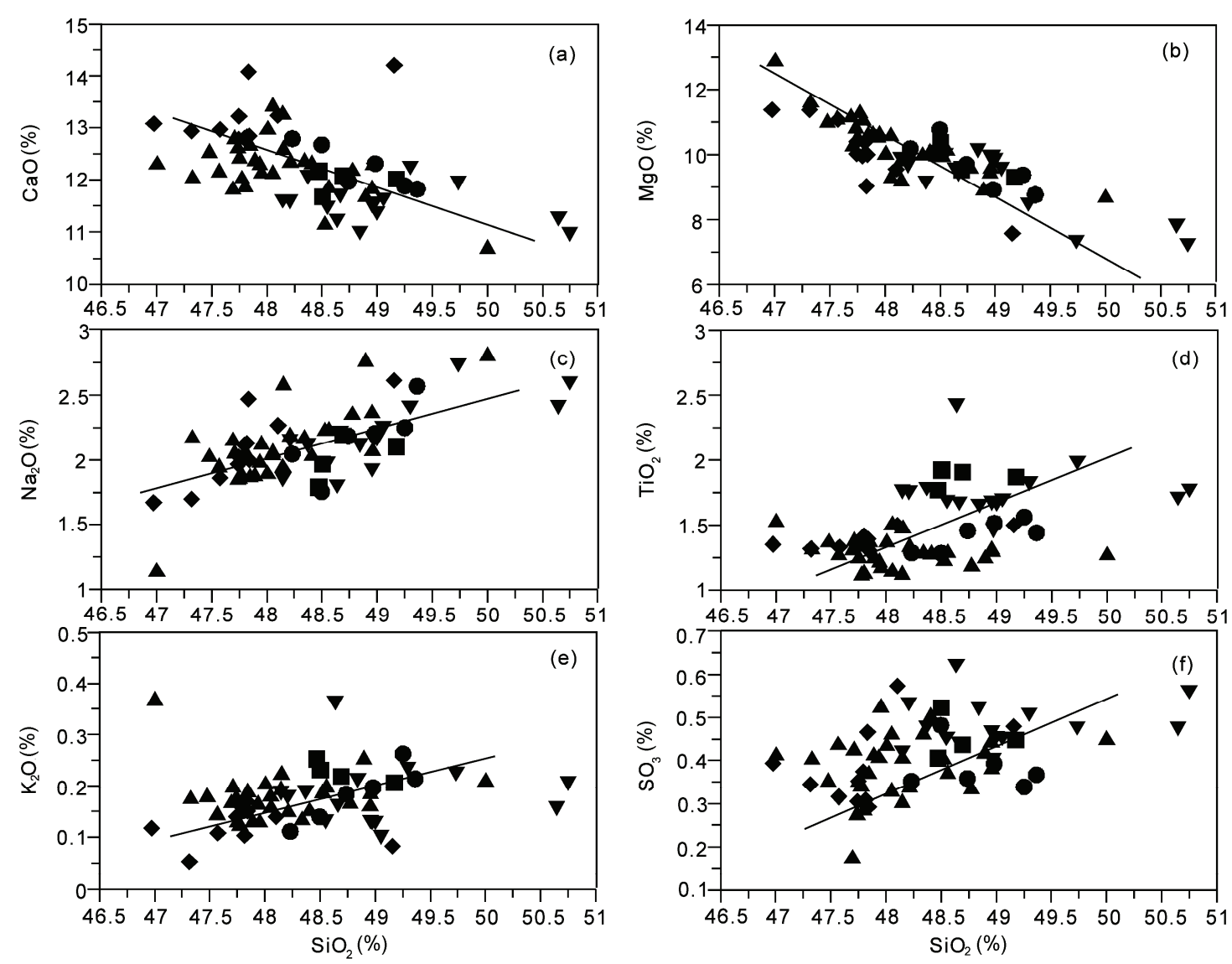

图 4 校正檄榄石分异结晶作用之后的初始熔体包裹体中 $\mathrm{SiO}_{2}$ 与 (a) $\mathrm{CaO},(\mathrm{b}) \mathrm{MgO},(\mathrm{c}) \mathrm{Na}_{2} \mathrm{O}$, (d) $\mathrm{TiO}_{2},(\mathrm{e}) \mathrm{K}_{2} \mathrm{O}$ 和(f) $\mathrm{SO}_{3}$ 之间 的相关性

图中符号与图 3(a)所示相同 
计算结果(网络附表 2)表明这些熔体包裹体平均结晶压 力为 $(0.83 \pm 0.25) \mathrm{GPa}$ (平均值士标准偏差), 对应于 24 $\mathrm{km}$ 的上地幔中. 这一结果远高于 Michael 和 Cornell ${ }^{[60]}$ 用同一压力计并根据东太平洋海隆玄武岩组成 $(\mathrm{MgO}$ $<8.0 \%$ )计算的岩浆结晶压力 $2.1 \sim 2.4 \mathrm{hPa}$ (大约为洋壳 内部的压力), 进一步说明这些橄榄石熔体包裹体代 表了岩浆在地幔中而不是洋壳中的演化端元.

\section{4 上地幔中的岩浆混合作用}

二辉橄榄岩熔融实验表明, 原始岩浆中的 $\mathrm{CaO} /$ $\mathrm{Al}_{2} \mathrm{O}_{3}$ 比值、 $\mathrm{Na}_{2} \mathrm{O}$ 含量分别与部分熔融程度呈良好正 相关和负相关, 而 $\mathrm{FeO}$ 含量与地幔熔融压力 (深度)呈 良好正相关 ${ }^{[36 ~ 38]}$. 但是，几乎所有的玄武质岩浆都 不是原始岩浆，而是经历了不同程度的分异结晶作 用, 而且不同分异结晶程度的洋中脊玄武岩均可构 成连续的演化性, 如, $\mathrm{MgO}$ 与 $\mathrm{CaO}, \mathrm{Al}_{2} \mathrm{O}_{3}, \mathrm{Na}_{2} \mathrm{O}$ 和 $\mathrm{FeO}$ 相关性很好. 因此, 这就可以将所有玄武岩根据 分异结晶趋势校正到 $\mathrm{MgO}=8 \mathrm{wt} \%$ 时的 $\mathrm{CaO}\left(\mathrm{Ca}_{8}\right)$, $\mathrm{Al}_{2} \mathrm{O}_{3}\left(\mathrm{Al}_{8}\right), \mathrm{Na}_{2} \mathrm{O}\left(\mathrm{Na}_{8}\right), \mathrm{FeO}\left(\mathrm{Fe}_{8}\right)$, 这样 $\mathrm{Ca}_{8} / \mathrm{Al}_{8}, \mathrm{Na}_{8}$ 和 $\mathrm{Fe}_{8}$ 分别可以替代原始岩浆中的 $\mathrm{CaO} / \mathrm{Al}_{2} \mathrm{O}_{3}, \mathrm{Na}_{2} \mathrm{O}$ 和 $\mathrm{FeO}$, 并分别用于计算和指示岩浆形成时的部分熔融 程度和深度 ${ }^{[35,37 \sim 40]}$. 为了解这些形成于地幔压力下 的熔体包裹体的形成与演化过程, 我们计算了能够 指示地幔源区部分熔融程度的 $\mathrm{Ca}_{8} / \mathrm{Al}_{8}, \mathrm{Na}_{8}$ 和指示地幔 熔融深度的 $\mathrm{Fe}_{8}$ (网络版附表 2). 很明显, $\mathrm{Na}_{8}-\mathrm{Ca}_{8} / \mathrm{Al}_{8}$
(图 6(a))与 $\mathrm{Na}_{8}-\mathrm{Fe}_{8}$ (图 6(b))均呈现出良好的负相关性, 说明越深的地幔源区 $\left(\mathrm{Fe}_{8}\right.$ 越高)温度越高, 因此岩浆 部分熔融程度越高 $\left(\mathrm{Na}_{8}\right.$ 越低), 也表明这些包裹体所 代表的玄武岩母岩浆端元来自于地幔源区不同熔融 程度和熔融深度. 据此可知, 地幔源区不同熔融条件 的玄武质岩浆在上升和通过地幔过程中经历了一定 的混合作用，这种混合作用的不同岩浆被伴随的结 晶橄榄石所捕获就形成了代表不同岩浆混合端元的 熔体包裹体.

在东太平洋海隆快速扩张中心, 由于较快的上 地幔减压速率，岩浆供应较强. 不同熔融条件的岩浆 上升迁移不是断续的和孤立的，而是在通过上地幔 过程中经历一定的混合作用. 为了更详细地了解这 些熔体包裹体所代表的不同岩浆端元对玄武岩组成 的贡献，我们对比分析了这些玄武岩和其中熔体包 裹体的 K/Ti 比, $\mathrm{Ca}_{8}, \mathrm{Al}_{8}, \mathrm{Fe}_{8}$ 和 $\mathrm{Na}_{8}$ 的组成范围和平 均值. 由图 7 可见, 这些值在寄主玄武岩中变化较小, 但在熔体包裹体中则具有很大的组成范围, 表明这 些玄武岩的母岩浆具有较强的组成多样性. 另外, 这 些包裹体和寄主玄武岩具有相似的平均 $\mathrm{K} / \mathrm{Ti}$ 比值, 但是, 就 $\mathrm{Ca}_{8}, \mathrm{Al}_{8}, \mathrm{Fe}_{8}$ 和 $\mathrm{Na}_{8}$ 而言, 包裹体相对寄主玄 武岩平均具有较低 $\mathrm{Ca}_{8}, \mathrm{Fe}_{8}$ 值和较高 $\mathrm{Al}_{8}, \mathrm{Na}_{8}$ 值, 因 此, 这些寄主玄武岩并不能由这些包裹体所代表的 母岩浆端元均匀混合而成. 因为一般认为玄武质岩 浆在洋中脊地壳中(尤其是在岩浆房内)经历了很强
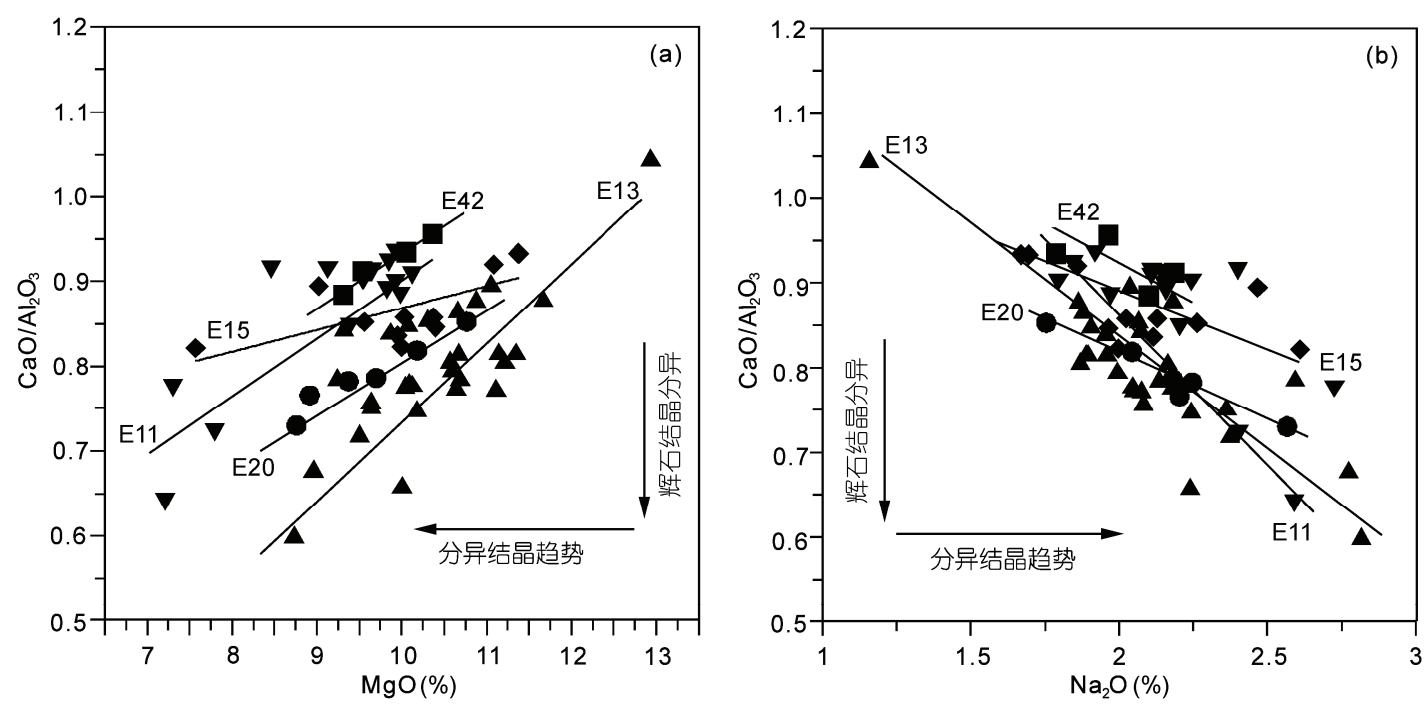

图 5 校正分异结晶作用后的初始熔体包裹体 $\mathrm{CaO} / \mathrm{Al}_{2} \mathrm{O}_{3}$ 比值与 (a) $\mathrm{MgO}$ 和(b) $\mathrm{Na}_{2} \mathrm{O}$ 的相关变化图 图中符号与图 3(a)所示相同 

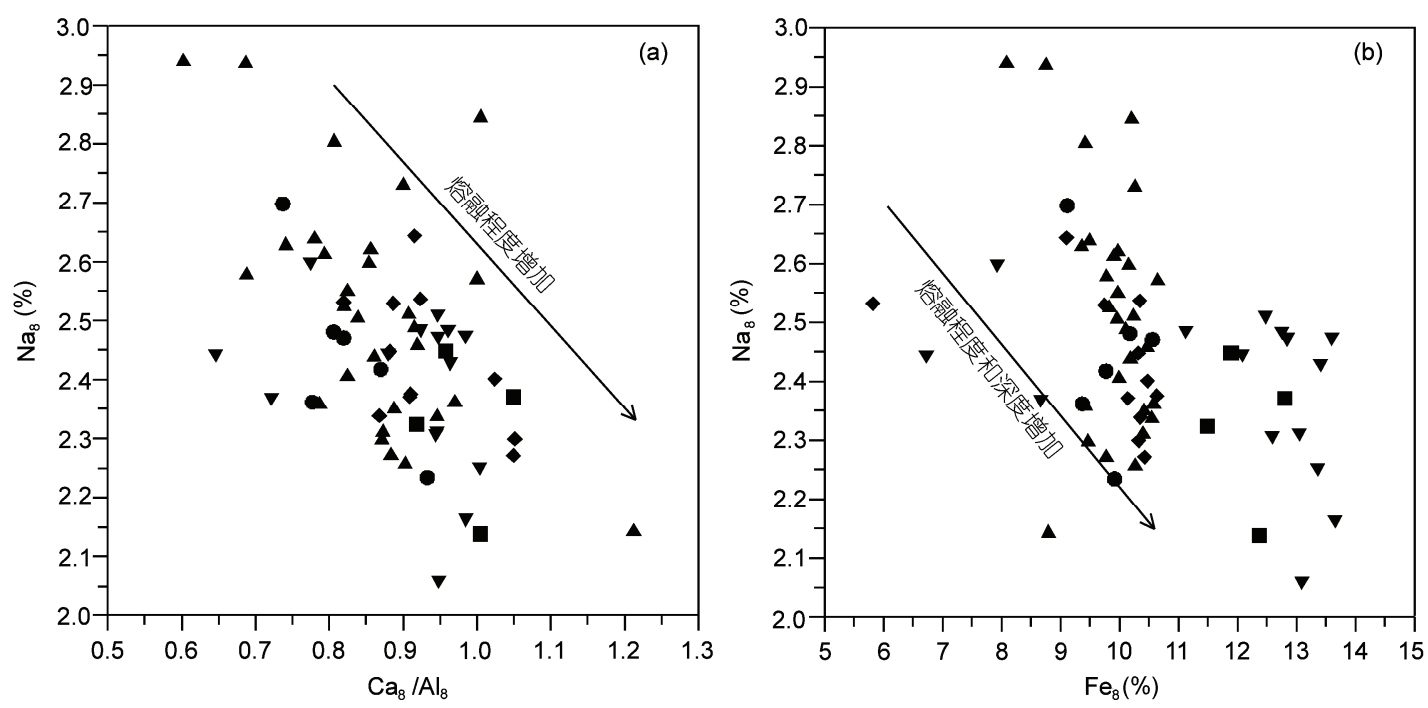

图 6 初始熔体包裹体内的 $\mathrm{Na}_{8}$ 与 (a) $\mathrm{Ca}_{8} / \mathrm{Al}_{8}$ 和(b) $\mathrm{Fe}_{8}$ 相关性图解 图中符号与图 3(a)所示相同

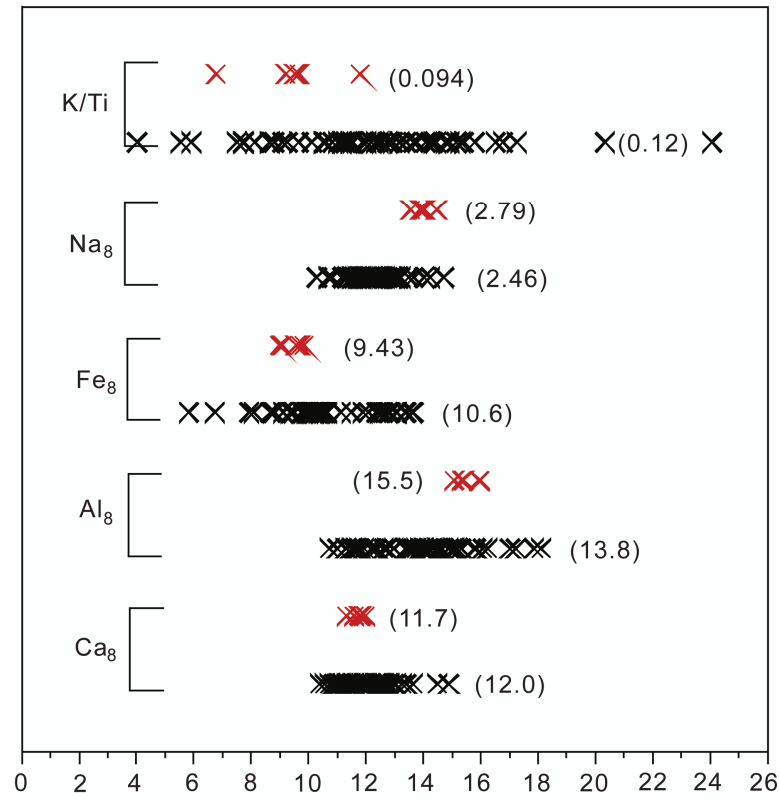

图 7 初始包裹体与寄主玄武岩的 $\mathrm{Ca}_{8}, \mathrm{Al}_{8}, \mathrm{Fe}_{8}, \mathrm{Na}_{8}(\%)$ 和 $\mathrm{K} / \mathrm{Ti}$ 变化范围对比图解

这些参数在包裹体中的变化范围明显高于其寄主玄武岩, 表明这些 包裹体代表了其寄主玄武岩在地幔中经历的不同混合作用端元

的混合和结晶作用, 我们推断这些玄武岩的化学组 成很大程度上也受洋壳内岩浆混合作用影响, 而这 些高 $\mathrm{Mg}$ \#橄榄石中的熔体包裹体代表的是不同端元 的岩浆在地幔中的混合作用(图 8).

玄武岩斑晶矿物内的熔体包裹体常被用于反映 岩浆在浅部洋壳内的混合作用, 但是本研究表明, 玄

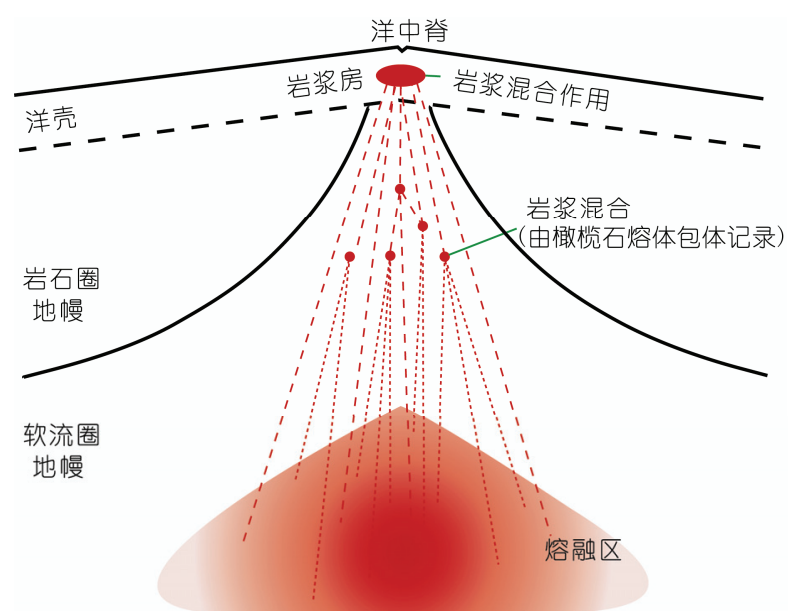

图 8 东太平洋海隆玄武质岩浆在地幔中经历岩浆混和和 结晶作用简图

武岩斑晶内熔体包裹体组成的多样性并不都反映岩 浆在洋壳内的混合和结晶作用. 玄武岩高 $\mathrm{Mg} \#$ 橄榄 石内的熔体包裹体可能常形成于地幔之中, 因此不 能与其他斑晶一起直接用于指示洋壳内岩浆低压演 化过程. 因此, 研究东太平洋海隆岩浆混合作用时, 也必须考虑不同端元的母岩浆在上地幔较高压力下 的混合作用对玄武岩组成的贡献.

\section{5 结论}

来自东太平洋海隆 $13^{\circ} \mathrm{N}$ 附近 5 个站位的玄武岩 具有很小的 $\mathrm{K} / \mathrm{Ti}, \mathrm{Tb} / \mathrm{Lu}, \mathrm{Sm} / \mathrm{Nd}$ 比值变化范围和相似 
的稀土元素配分型式, 前人在研究区获得的 $\mathrm{Sr}$ 和 $\mathrm{Nd}$ 同位素组成变化范围也很小, 均表明研究区下部地 幔源区不均一性很弱. 通过薄片电子探针分析获得 了 65 个高 $\mathrm{Mg} \#$ 橄榄石内熔体包裹体主量元素组成, 并通过校正包裹体的后期橄榄石分异结晶作用获得 了初始熔体包裹体组成. 结果表明:

(1) 这些包裹体均具有较寄主玄武岩高的 $\mathrm{MgO}$ 含 量, 可以代表寄主岩浆较早期不同演化阶段, 并可以 指示岩浆较早期阶段的演化过程.

(2) 玄武岩薄片内只出现橄榄石和斜长石斑晶、 全岩 $\mathrm{CaO} / \mathrm{Al}_{2} \mathrm{O}_{3}-\mathrm{MgO}$ 负相关的事实和相平衡计算均 表明, 岩浆在低压岩浆房内未结晶出单斜辉石. 但是, 熔体包裹体中 $\mathrm{CaO} / \mathrm{Al}_{2} \mathrm{O}_{3}-\mathrm{MgO}$ 正相关性、 $\mathrm{CaO} / \mathrm{Al}_{2} \mathrm{O}_{3}$ $\mathrm{Na}_{2} \mathrm{O}$ 负相关性以及较寄主玄武岩很高的 $\mathrm{CaO} / \mathrm{Al}_{2} \mathrm{O}_{3}$ 比值, 均表明这些寄主玄武岩经历了较高压力下的 单斜辉石分异. 玄武质岩浆在较深部位结晶出单斜
辉石, 但随着岩浆上升至洋壳过程中的压力减小, 单 斜辉石逐渐偏离液相线. 根据单斜辉石对结晶压力 的依赖性，计算获得 65 个熔体包裹体平均结晶(捕获) 压力为 $(0.83 \pm 0.25) \mathrm{GPa}$ (平均值土标准偏差). 可见, 这 些橄榄石熔体包裹体平均被捕获于洋底以下 24 km 的上地幔中.

(3) 初始熔体包裹体的 $\mathrm{Ca}_{8} / \mathrm{Al}_{8}, \mathrm{Na}_{8}$ 与 $\mathrm{Fe}_{8}$ 之间的 关系，表明这些熔体包裹体形成于相同组成上地幔的 不同熔融程度和深度. 初始熔体包裹体 $\mathrm{Ca}_{8} / \mathrm{Al}_{8}, \mathrm{Na}_{8}$, $\mathrm{Fe}_{8}$ 和 $\mathrm{K} / \mathrm{Ti}$ 比的变化范围均明显高于寄主玄武岩，显示 玄武质岩浆在通过深部地幔过程中经历了形成于不同 熔融程度和深度的岩浆混合作用. 因此, 形成于上地 幔压力下的高 $\mathrm{Mg} \#$ 橄榄石内熔体包裹体不能与其他斑 晶中的熔体包裹体共同用于指示洋壳内的岩浆过程, 即在研究玄武质岩浆混合作用时，必须考虑形成于不 同熔融条件的岩浆在上地幔中经历的混合作用.

致谢 感谢参加 DY105-12, 14 航次的所有队员为样品的获得所做的贡献; 感谢中国地质科学院地质研究所周剑雄、余静 在本研究中的电子探针分析上给予的帮助.

\section{参考文献}

1 Stolper E. A phase diagram for mid-ocean ridge basalts: Preliminary results and implications for petrogenesis. Contrib Mineral Petrol, 1980, 74: 13-27

2 Blake S, Rogers N. Magma differentiation rates from $\left({ }^{226} \mathrm{Ra} /{ }^{230} \mathrm{Th}\right)$ and the size and power out put of magma chambers. Earth Planet Sci Lett, 2005, 236: 654-669

3 Kinzler R, Grove T. Primary magmas of mid-ocean ridge basalt 2. applications. J Geophys Res, 1992, 97: 6907—6926

4 Sinton J M, Detrick R S. Mid-ocean ridge magma chambers. J Geophys Res, 1992, 97: 197-216

5 Zhang S M, Wang F Z. Basalts action on research geosphere deep course and structural setting (in Chinese). Adv Earth Sci, 2002, 17: $685-692$

6 Herzberg C. Partial crystallization of mid-ocean ridge basalts in the crust and mantle. J Petrol, 2004, 45: 2389-2405

7 Rubin K H, Sinton J M. Inferences on mid-ocean ridge thermal and magmatic structure from MORB compositions. Earth Planet Sci Lett, 2007, 260: 257-276

8 Bach W, Hegner E, Erzinger J, et al. Chemical and isotopic variations along the superfast spreading East Pacific Rise from $6^{\circ}$ to $30^{\circ} \mathrm{S}$ Contrib Mineral Petrol, 1994, 116: 365-380

9 郑建平. 不同时空背景幔源物质对比华北深部岩石圈破坏和增生置换过程. 科学通报, 2009, 54: 1990-2007

10 Sleep N H. Tapping of Magmas from Ubiquitous Mantle Heterogeneities: An Alternative to Mantle Plumes? J of Geophys Res, 1984, 89: $10029-10041$

11 Kelemen P B, Koga K, Shimizu N. Geochemistry of gabbro sills in the crust-mantle transition zone of the Oman ophiolite: Implications for the origin of the oceanic lower crust. Earth Planet Sci Lett, 1997, 146: 475—488

12 Wagner T P, Grove T L. Melt/harzburgite reaction in the petrogenesis of tholeiitic magma from Kilauea volcano, Hawaii. Contrib Mineral Petrol, 1998, 131: 1-12

13 Stevenson D S, Blake S. Modelling the dynamics and thermodynamics of volcanic degassing. Bull Volcanol, 1998, 60: 307-317

14 Kelemen P B, Dick H J B. Focused melt flow and localized deformation in the upper mantle: Juxtaposition of replacive dunite and ductile shear zones in the Josephine peridotites, SW Oregon. J Geophys Res, 1995, 100: 423—438

15 Kelemen P B, Shimizu N, Salters V J M. Focussed flow of melt in the upper mantle: Extraction of MORB beneath oceanic spreading 
ridges. Mineral Mag, 1994, 58: 466-467

16 Scarrow J H, Cox K G. Basalts generated by decompressive adiabatic melting of a mantle plume: A case study from the Isle of Skye, NW Scotland. J Petrol, 1995, 36: 3-22

17 Sleep N H. Hotspot Volcanism and Mantle Plumes. Annu Rev Earth Planet Sci, 1992, 20: 19—43

18 Niu Y L, Langmuir C H, Kinzler R J, The origin of abyssal peridotites: A new perspective. Earth Planet Sci Lett, 1997, 152 : 251-265

19 Anderson A T. Magma mixing: Petrological processes and volcanological tools. J Volcanol Geoth Res, 1976, 1: 3-33

20 Dungan M A, Rhodes J M. Residual Glasses and Melt Inclusions in Basalts From DSDP Legs 45 and 46 : Evidence for Magma Mixing. Contrib Mineral Petrol, 1978, 67: 417-431

21 Sobolev A V. Melt inclusions in minerals as a source of principal petrologic information. Petrol, 1996, 4: 228-239

22 Gaetani G A, Watson E B. Open system behavior of olivine-hosted melt inclusions. Earth Planet Sci Lett, 2000, 183: 27-41

23 Schiano P. Primitive mantle magmas recorded as silicate melt inclusions in igneous minerals. Earth-Sci Rev, 2003, 63: 121-144

24 Eason D, Sinton J. Origin of high-Al N-MORB by fractional crystallization in the upper mantle beneath the Galápagos Spreading Center. Earth Planet Sci Lett, 2006, 252: 423-436

25 Falloon T J, Danyushevsky L V, Ariskin A, et al. The application of olivine geothermometry to infer crystallization temperatures of parental liquids: Implications for the temperature of MORB magmas. Chem Geol, 2007, 241: 207-233

26 Sours-Page R, Nielsen R L, Batiza R. Melt inclusions as indicators of parental magma diversity on the northern East Pacific Rise. Chem Geol, 2002, 183: 237-261

27 Choukroune P, Francheteau J, Hekinian R. Tectonics of the East Pacific Rise near $12^{\circ} 50^{\prime}$ N: A submersible study. Earth Planet Sci Lett, 1984, 68: 115-127

28 Ballard R D, Hekinian R, Francheteau J. Geological setting of hydrothermal activity at $12^{\circ} 50$ 'N on the East Pacific Rise: A submersible study. Earth Planet Sci Lett, 1984, 69: 176-186

29 曾志刚, 王晓媛, 张国良, 等. 东太平洋海隆 $13^{\circ} \mathrm{N}$ 附近 $\mathrm{Fe}$-氧差化物的形成: 矿物和地球化学证据. 中国科学 $\mathrm{D}$ 辑: 地球科学, 2007 , 37: $1349-1357$

30 张国良, 曾志刚, 殷学博, 等. 东太平洋海隆 $13^{\circ} \mathrm{N}$ 附近岩浆周期性混合作用: 模拟计算和环带斜长石证据. 中国科学 $\mathrm{D}$ 辑: 地球科 学, 2009, 39: 35-50

31 Sakai H, Marais D J D, Ueda A, et al. Concentrations and isotope ratios of carbon, nitrogen and sulfur in ocean-floor basalts. Geoch Cosmoch Acta, 1984, 48: 2433-2441

32 Saal A E, Hauri E H, Langmuir C H, et al. Vapour undersaturation in primitive mid-ocean-ridge basalt and the volatile content of Earth's upper mantle. Nature, 2002, 419: 451—455

33 le Roux P J, Shirey S B, Hauri E H, et al. The effects of variable sources, processes and contaminants on the composition of northern EPR MORB $\left(8^{\circ}-10^{\circ} \mathrm{N}\right.$ and $\left.12^{\circ}-14^{\circ} \mathrm{N}\right)$ : Evidence from volatiles $\left(\mathrm{H}_{2} \mathrm{O}, \mathrm{CO}_{2}, \mathrm{~S}\right)$ and halogens $(\mathrm{F}, \mathrm{Cl})$. Earth Planet Sci Lett, 2006, 251: $209-231$

34 Ford C E, Russell D G, Craven J A, et al. Olivine-liquid equilibria: Temperature, pressure and composition dependence of the crystal/liquid cation partition coefficients for $\mathrm{Mg}, \mathrm{Fe}^{2+}$, Ca and Mn. J Petrol, 1983, 24: 256-265

35 Taylor B, Martinez F. Back-arc basin basalt systematics. Earth Planet Sci Lett, 2003, 210: 481-497

36 Ligi M, Bonatti E, Cipriani A, et al. Water-rich basalts at mid-oceanridge cold spots. Nature, 2005, 434: 66-69

37 Niu Y L, Batiza R. An empirical method for calculating melt compositions produced beneath mid-ocean ridges: Application for axis and off-axis (seamounts) melting. J Geophys Res, 1991, 96: 21753-21777

38 Niu Y L, Waggoner D G, Sinton J M, et al. Mantle source heterogeneity and melting processes beneath seafloor spreading centres: The East Pacific Rise, $18^{\circ}-19^{\circ}$ S. J Geophys Res, 1996, 101: 27711-27733

39 Hékiniana R, Francheteaub J, Armijoc R, et al. Petrology of the Easter microplate region in the South Pacific. J Volcanol Geoth Res, 1996, 72: $259-289$

40 Hirose K, Kushiro I. Partial melting of dry peridotites at high pressures: Determination of compositions of melts segregated from peridotite using aggregates of diamond. Earth Planet Sci Lett, 1993, 114: 477—489

41 Macdougall J D, Lugmair G W. Sr and Nd isotopes in basalts from the East Pacific Rise: Significance for mantle heterogeneity. Earth Planet Sci Lett, 1986, 77: 273-284

42 Niu Y L, Regelous M, Wendt I J, et al. Geochemistry of near-EPR seamounts: Importance of source vs. process and the origin of enriched mantle component. Earth Planet Sci Lett, 2002, 199: 327-345

43 Castillo $\mathrm{P} \mathrm{R}$, Klein E, Bender $\mathrm{J}$, et al. Petrology and $\mathrm{Sr}$, $\mathrm{Nd}$, and $\mathrm{Pb}$ isotope geochemistry of mid-ocean ridge basalt glasses from the $11^{\circ} 45^{\prime} \mathrm{N}$ to $15^{\circ} 00^{\prime} \mathrm{N}$ segment of the East Pacific Rise. Geochem Geophys Geosys, 2000, doi: 10.1029/1999GC000024

44 Chaussidon M, Sheppard S M F, Hydrogen M A. Hydrogen, sulphur and neodymium isotope variations in the mantle beneath the EPR at 
$12^{\circ} 50^{\prime}$ N. J Geochem Soc, 1991, 3: 325-337

45 Hamelin B, Dupper B, Allegre C J. Lead-Strontium isotopic variations along the East Pacific Rise and the mid-Atlantic ridge: A comparative study. Earth Planet Sci Lett, 1984, 67: 340-350

46 Caroff M, Fleutelot C. The north-south propagating spreading center of the North Fiji Basin: Modeling of the geochemical evolution in periodically replenished and tapped magma chambers. Mineral Petrol, 2003, 79: 203-224

47 Danyushevsky L V, McNeill A W, Sobolev A V. Experimental and petrological studies of melt inclusions in phenocrysts from mantle-derived magmas: An overview of techniques, advantages and complications. Chem Geol, 2002, 183: 5—24

48 Kress V C, Ghiorso M S. Thermodynamic modelling of post-entrapment crystallization in igneous phases. J Volcanol Geoth Res, 2004, 137: 247-260

49 Danyushevsky L V. The effect of small amounts of $\mathrm{H}_{2} \mathrm{O}$ on crystallisation of mid-ocean ridge and backarc basin magmas. J Volcanol Geoth Res, 2001, 110: 265-280

50 Danyushevsky L V, Della-Pasqua F N, Sokolov S. Re-equilibration of melt inclusions trapped by magnesian olivine phenocrysts from subduction-related magmas: Petrological implications. Contrib Mineral Petrol, 2000, 138: 68-83

51 Zhang G L, Zeng Z G, Yin X B, et al. Deep fractionation of clinopyroxene in the East Pacific Rise $13^{\circ} \mathrm{N}$ : Evidence from high MgO MORB and melt inclusions. Acta Geol Sin-Engl, 2009, 83: 266-277

52 Gaillard F, Scaillet B, Pichavant M, et al. The effect of water and $\mathrm{fO}_{2}$ on the ferric-ferrous ratio of silicic melts. Chem Geol, 2001, 174: $255-273$

53 Ulmer P. Partial melting in the mantle wedge- - The role of $\mathrm{H}_{2} \mathrm{O}$ in the genesis of mantle-derived 'arc-related' magmas. Phys Earth Planet Int, 2001, 127: 215-232

54 Müntener $\mathrm{O}$, Kelemen $\mathrm{P}$ B, Grove T L. The role of $\mathrm{H}_{2} \mathrm{O}$ during crystallization of primitive arc magmas under uppermost mantle conditions and genesis of igneous pyroxenites: An experimental study. Contrib Mineral Petrol, 2001, 141: 643-658

55 Medard E, McCammon C A, Barr J A, et al. Oxygen fugacity, temperature reproducibility, and $\mathrm{H}_{2} \mathrm{O}$ contents of nominally anhydrous piston-cylinder experiments using graphite capsules. Am Mineral, 2008, 93: 1838-1844

56 Berndt J, Koepke J, Holtz F. An experimental investigation of the influence of water and oxygen fugacity on differentiation of MORB at 200 MPa. J Petrol, 2005, 46: 135-167

57 Michael P J, Chase R L. The influence of primary magma composition, $\mathrm{H}_{2} \mathrm{O}$ and pressure on Mid-Ocean Ridge basalt differentiation. Contrib Mineral Petrol, 1987, 96: 245-263

58 Iyer S D, Ray D. Structure, tectonic and petrology of mid-oceanic ridges and the Indian scenario. Curr Sci, 2003, 85: 277-289

59 Yang H J, Kinzler R J, Grove T L. Experiments and models of anhydrous basaltic olivine-plagioclase-augite saturated melts from 0.001 to 10 kbar. Contrib Mineral Petrol, 1996, 124: 1-18

60 Michael P J, Cornell W C. Influence of spreading rate and magma supply on crystallization and assimilation beneath midocean ridges: Evidence from chlorine and major element chemistry of mid-ocean ridge basalts. J Geophys Res, 1998, 103: 18325—18356

61 Yang H J, Frey F A, Clague D A, et al. Mineral chemistry of submarine lavas from Hilo Ridge, Hawaii: Implications for magmatic processes within Hawaiian rift zones. Contrib Mineral Petrol, 1999, 135: 355-372

62 le Roux P J, le Roex A P, Schilling J G. Crystallization processes beneath the southern Mid-Atlantic Ridge $\left(40^{\circ}-55^{\circ} \mathrm{S}\right)$, evidence for high-pressure initiation of crystallization. Contrib Mineral Petrol, 2002, 142: 582—602

63 Wallace P J. Volatiles in submarine basaltic glasses from the Northern Kerguelen Plateau (ODP Site 1140): Implications for source region compositions, magmatic processes, and plateau subsidence. J Petrol, 2002, 43: 1311-1326

64 Villiger S, Ulmer P, Müntener O. Equilibrium and fractional crystallization experiments at $0.7 \mathrm{GPa}$ : The effect of pressure on phase relations and liquid compositions of tholeiitic Magmas. J Petrol, 2007, 48: 159—184

65 Kelley D F, Barton M. Pressures of crystallization of icelandic magmas. J Petrol, 2008, 49: 465—492 
附表 1 橄榄石熔体包裹体主量元素组成 $(\%)$ 和主矿物橄榄石 $\mathrm{Mg} \#\left(100 \times \mathrm{Mg}^{2+} /\left(\mathrm{Fe}^{2+}+\mathrm{Mg}^{2+}\right)\right)$

\begin{tabular}{|c|c|c|c|c|c|c|c|c|c|c|c|c|c|c|c|c|c|}
\hline 包裹体 & $\mathrm{SiO}_{2}$ & $\mathrm{TiO}_{2}$ & $\mathrm{Al}_{2} \mathrm{O}_{3}$ & $\mathrm{FeO}_{\mathrm{t}}$ & $\mathrm{CaO}$ & $\mathrm{MnO}$ & $\mathrm{MgO}$ & $\mathrm{Na}_{2} \mathrm{O}$ & $\mathrm{K}_{2} \mathrm{O}$ & $\mathrm{P}_{2} \mathrm{O}_{5}$ & $\mathrm{Cr}_{2} \mathrm{O}_{3}$ & $\mathrm{SO}_{3}$ & Total & $\mathrm{K} / \mathrm{Ti}$ & $\mathrm{CaO} / \mathrm{Al}_{2} \mathrm{O}_{3}$ & $\begin{array}{c}\text { 平衡 } \\
\mathrm{Mg \#}^{\mathrm{a})}\end{array}$ & $\begin{array}{l}\text { 主矿物 } \\
\mathrm{Mg \#}^{\mathrm{b})}\end{array}$ \\
\hline E42-1 & 48.8 & 1.97 & 14.5 & 10.1 & 13.5 & 0.14 & 6.15 & 1.99 & 0.28 & 0.11 & 0.09 & 0.45 & 98.1 & 0.14 & 0.94 & 76.7 & 84.2 \\
\hline E42-2 & 49.3 & 2.25 & 14.3 & 10.1 & 13.6 & 0.16 & 4.75 & 2.30 & 0.27 & 0.21 & 0.09 & 0.61 & 98.0 & 0.12 & 0.95 & 71.7 & 84.0 \\
\hline E42-3 & 49.6 & 2.19 & 15.2 & 9.40 & 13.8 & 0.18 & 4.73 & 2.51 & 0.25 & 0.15 & 0.15 & 0.50 & 98.7 & 0.11 & 0.91 & 73.1 & 84.1 \\
\hline E42-4 & 49.7 & 2.09 & 15.2 & 9.47 & 13.4 & 0.13 & 5.06 & 2.35 & 0.23 & 0.14 & 0.11 & 0.50 & 98.4 & 0.11 & 0.89 & 74.3 & 84.0 \\
\hline E20-1 & 48.7 & 1.39 & 16.1 & 8.52 & 13.7 & 0.12 & 7.60 & 1.90 & 0.15 & 0.01 & 0.21 & 0.52 & 98.9 & 0.11 & 0.85 & 82.8 & 88.0 \\
\hline E20-2 & 48.4 & 1.39 & 16.9 & 8.35 & 13.8 & 0.15 & 6.87 & 2.21 & 0.12 & 0.23 & 0.13 & 0.38 & 98.9 & 0.09 & 0.82 & 81.6 & 88.0 \\
\hline E20-3 & 50.2 & 1.78 & 18.9 & 7.25 & 14.4 & 0.13 & 2.68 & 2.59 & 0.23 & 0.18 & 0.21 & 0.46 & 98.9 & 0.13 & 0.76 & 66.6 & 86.2 \\
\hline E20-4 & 49.9 & 1.75 & 18.3 & 7.65 & 14.3 & 0.10 & 2.59 & 2.62 & 0.22 & 0.16 & 0.17 & 0.43 & 98.2 & 0.13 & 0.78 & 64.6 & 86.2 \\
\hline E20-5 & 50.7 & 1.69 & 19.0 & 7.17 & 13.8 & 0.07 & 2.64 & 3.01 & 0.25 & 0.18 & 0.23 & 0.43 & 99.1 & 0.15 & 0.73 & 66.5 & 86.2 \\
\hline E20-6 & 50.3 & 1.85 & 18.0 & 7.50 & 14.0 & 0.07 & 2.67 & 2.66 & 0.31 & 0.16 & 0.16 & 0.40 & 98.0 & 0.17 & 0.78 & 65.8 & 86.1 \\
\hline E15-1 & 48.8 & 1.56 & 18.7 & 7.56 & 15.3 & 0.21 & 3.00 & 2.39 & 0.18 & 0.06 & 0.13 & 0.35 & 98.3 & 0.12 & 0.82 & 68.2 & 87.1 \\
\hline E15-2 & 48.7 & 1.61 & 18.3 & 7.85 & 15.4 & 0.03 & 2.90 & 2.38 & 0.17 & 0.11 & 0.15 & 0.37 & 97.9 & 0.11 & 0.84 & 66.6 & 87.1 \\
\hline E15-3 & 49.0 & 1.63 & 18.8 & 5.99 & 15.4 & 0.05 & 3.43 & 2.58 & 0.09 & 0.21 & 0.14 & 0.52 & 97.9 & 0.06 & 0.82 & 75.6 & 87.1 \\
\hline E15-4 & 48.8 & 1.70 & 18.4 & 7.45 & 15.3 & 0.28 & 2.84 & 2.54 & 0.19 & 0.21 & 0.21 & 0.45 & 98.4 & 0.11 & 0.83 & 67.3 & 87.1 \\
\hline E15-5 & 47.7 & 1.62 & 16.8 & 8.60 & 15.6 & 0.12 & 4.62 & 2.00 & 0.14 & 0.00 & 0.40 & 0.47 & 98.1 & 0.09 & 0.93 & 74.4 & 87.5 \\
\hline E15-6 & 47.8 & 1.48 & 15.7 & 8.98 & 14.4 & 0.18 & 6.95 & 2.07 & 0.12 & 0.00 & 0.20 & 0.35 & 98.3 & 0.08 & 0.92 & 80.7 & 87.5 \\
\hline E15-7 & 47.7 & 1.49 & 15.7 & 9.09 & 14.6 & 0.24 & 6.77 & 1.92 & 0.06 & 0.15 & 0.25 & 0.39 & 98.3 & 0.04 & 0.93 & 80.1 & 87.5 \\
\hline E15-8 & 48.5 & 1.73 & 17.9 & 7.41 & 15.2 & 0.18 & 3.52 & 2.61 & 0.16 & 0.16 & 0.08 & 0.66 & 98.1 & 0.09 & 0.85 & 72.0 & 87.4 \\
\hline E15-9 & 48.0 & 1.56 & 17.6 & 7.19 & 15.7 & 0.12 & 4.23 & 2.76 & 0.19 & 0.11 & 0.19 & 0.52 & 98.1 & 0.12 & 0.89 & 76.1 & 87.4 \\
\hline E15-10 & 47.7 & 1.47 & 16.0 & 8.74 & 13.7 & 0.19 & 7.44 & 2.28 & 0.11 & 0.12 & 0.21 & 0.33 & 98.3 & 0.07 & 0.85 & 82.1 & 87.5 \\
\hline E15-11 & 48.7 & 1.61 & 18.5 & 7.10 & 15.8 & 0.21 & 2.97 & 2.43 & 0.20 & 0.08 & 0.18 & 0.42 & 98.1 & 0.12 & 0.85 & 69.3 & 87.5 \\
\hline E13-1 & 48.5 & 1.67 & 16.9 & 8.50 & 15.1 & 0.16 & 4.02 & 2.46 & 0.22 & 0.22 & 0.20 & 0.43 & 98.4 & 0.13 & 0.89 & 71.9 & 87.3 \\
\hline E13-2 & 49.1 & 1.67 & 17.6 & 8.04 & 15.4 & 0.17 & 3.50 & 2.28 & 0.22 & 0.15 & 0.15 & 0.34 & 98.6 & 0.13 & 0.87 & 70.2 & 87.3 \\
\hline E13-3 & 48.8 & 2.04 & 15.6 & 9.12 & 16.2 & 0.14 & 3.32 & 1.53 & 0.49 & 0.10 & 0.20 & 0.55 & 98.1 & 0.24 & 1.04 & 66.3 & 87.3 \\
\hline E13-4 & 48.1 & 1.70 & 17.8 & 7.56 & 15.0 & 0.17 & 4.28 & 2.32 & 0.18 & 0.10 & 0.17 & 0.52 & 97.9 & 0.11 & 0.84 & 75.4 & 87.3 \\
\hline E13-5 & 48.3 & 1.59 & 17.1 & 8.37 & 14.6 & 0.17 & 5.18 & 2.36 & 0.23 & 0.15 & 0.19 & 0.49 & 98.6 & 0.14 & 0.85 & 77.0 & 87.3 \\
\hline E13-6 & 48.3 & 1.60 & 16.9 & 8.44 & 14.6 & 0.16 & 4.93 & 2.17 & 0.22 & 0.09 & 0.15 & 0.43 & 98.0 & 0.14 & 0.86 & 75.9 & 87.3 \\
\hline E13-7 & 48.1 & 1.55 & 17.1 & 8.39 & 14.5 & 0.21 & 5.19 & 2.13 & 0.23 & 0.00 & 0.14 & 0.49 & 98.0 & 0.15 & 0.85 & 77.0 & 87.3 \\
\hline E13-8 & 48.7 & 1.72 & 18.5 & 7.30 & 14.5 & 0.14 & 3.29 & 2.99 & 0.26 & 0.22 & 0.22 & 0.47 & 98.3 & 0.15 & 0.79 & 70.9 & 87.3 \\
\hline E13-9 & 48.4 & 1.37 & 18.7 & 8.16 & 12.3 & 0.17 & 5.25 & 2.47 & 0.21 & 0.10 & 0.19 & 0.45 & 97.8 & 0.15 & 0.66 & 77.6 & 87.7 \\
\hline E13-10 & 49.0 & 1.46 & 18.1 & 7.63 & 13.7 & 0.13 & 4.53 & 2.32 & 0.21 & 0.12 & 0.13 & 0.43 & 97.7 & 0.14 & 0.76 & 76.2 & 87.7 \\
\hline E13-11 & 49.4 & 1.33 & 18.5 & 7.43 & 11.1 & 0.12 & 6.27 & 2.92 & 0.22 & 0.09 & 0.11 & 0.47 & 97.9 & 0.17 & 0.60 & 82.0 & 87.7 \\
\hline E13-12 & 48.3 & 1.43 & 17.4 & 8.36 & 13.0 & 0.14 & 5.56 & 2.46 & 0.22 & 0.08 & 0.17 & 0.41 & 97.6 & 0.15 & 0.75 & 78.2 & 87.8 \\
\hline E13-13 & 48.2 & 1.43 & 17.2 & 8.83 & 14.0 & 0.10 & 5.50 & 2.14 & 0.15 & 0.16 & 0.12 & 0.47 & 98.3 & 0.10 & 0.81 & 77.1 & 87.4 \\
\hline E13-14 & 48.1 & 1.32 & 16.6 & 9.09 & 13.2 & 0.15 & 7.69 & 2.14 & 0.18 & 0.15 & 0.13 & 0.44 & 99.1 & 0.14 & 0.79 & 82.0 & 87.7 \\
\hline E13-15 & 49.4 & 1.64 & 18.2 & 8.49 & 14.6 & 0.05 & 3.47 & 2.68 & 0.21 & 0.11 & 0.22 & 0.22 & 99.3 & 0.13 & 0.80 & 68.8 & 87.7 \\
\hline E13-16 & 48.1 & 1.48 & 17.2 & 9.06 & 14.0 & 0.14 & 5.42 & 2.26 & 0.17 & 0.18 & 0.07 & 0.51 & 98.6 & 0.11 & 0.81 & 76.4 & 87.7 \\
\hline E13-17 & 48.0 & 1.57 & 16.2 & 9.32 & 14.2 & 0.18 & 5.27 & 2.58 & 0.21 & 0.12 & 0.13 & 0.48 & 98.3 & 0.13 & 0.88 & 75.3 & 87.6 \\
\hline E13-18 & 48.0 & 1.50 & 17.6 & 8.15 & 13.7 & 0.15 & 5.04 & 2.44 & 0.17 & 0.09 & 0.11 & 0.38 & 97.3 & 0.11 & 0.78 & 77.0 & 87.7 \\
\hline E13-19 & 47.9 & 1.35 & 17.6 & 8.35 & 14.3 & 0.11 & 3.72 & 2.26 & 0.15 & 0.15 & 0.14 & 0.41 & 96.5 & 0.11 & 0.81 & 70.6 & 87.7 \\
\hline E13-20 & 48.7 & 1.35 & 17.6 & 8.84 & 13.8 & 0.14 & 5.61 & 2.43 & 0.15 & 0.13 & 0.13 & 0.60 & 99.5 & 0.11 & 0.79 & 77.4 & 87.8 \\
\hline E13-21 & 48.3 & 1.42 & 17.4 & 8.29 & 13.5 & 0.17 & 5.93 & 2.38 & 0.15 & 0.17 & 0.08 & 0.51 & 98.3 & 0.11 & 0.78 & 79.4 & 87.8 \\
\hline E13-22 & 48.5 & 1.28 & 17.3 & 8.21 & 13.0 & 0.16 & 6.27 & 2.52 & 0.18 & 0.18 & 0.14 & 0.36 & 98.1 & 0.14 & 0.75 & 80.5 & 87.7 \\
\hline E13-23 & 47.9 & 1.42 & 17.4 & 8.76 & 14.0 & 0.14 & 5.36 & 2.11 & 0.15 & 0.12 & 0.21 & 0.40 & 98.0 & 0.11 & 0.81 & 76.8 & 87.4 \\
\hline E13-24 & 48.6 & 1.43 & 17.5 & 8.43 & 13.6 & 0.06 & 5.74 & 2.26 & 0.17 & 0.15 & 0.12 & 0.56 & 98.6 & 0.12 & 0.77 & 78.6 & 87.6 \\
\hline E13-25 & 48.7 & 1.46 & 18.1 & 7.68 & 13.0 & 0.14 & 4.74 & 2.61 & 0.18 & 0.22 & 0.13 & 0.49 & 97.4 & 0.12 & 0.72 & 76.9 & 87.6 \\
\hline E13-26 & 48.8 & 1.38 & 18.9 & 7.44 & 12.8 & 0.10 & 4.48 & 3.04 & 0.28 & 0.12 & 0.10 & 0.46 & 97.8 & 0.20 & 0.68 & 76.5 & 87.5 \\
\hline E13-27 & 49.2 & 1.42 & 19.1 & 7.73 & 14.7 & 0.12 & 2.81 & 2.58 & 0.19 & 0.17 & 0.10 & 0.36 & 98.5 & 0.13 & 0.77 & 66.3 & 88.0 \\
\hline E13-28 & 48.8 & 1.39 & 18.8 & 7.53 & 14.5 & 0.13 & 3.18 & 2.46 & 0.22 & 0.18 & 0.17 & 0.40 & 97.6 & 0.16 & 0.77 & 69.5 & 88.0 \\
\hline E13-29 & 49.1 & 1.33 & 18.5 & 7.47 & 15.5 & 0.07 & 3.67 & 2.29 & 0.23 & 0.16 & 0.17 & 0.36 & 98.9 & 0.17 & 0.84 & 72.6 & 87.7 \\
\hline E11-1 & 49.4 & 2.09 & 15.5 & 9.72 & 14.1 & 0.21 & 3.65 & 2.48 & 0.22 & 0.08 & 0.19 & 0.56 & 98.2 & 0.11 & 0.91 & 67.0 & 82.1 \\
\hline E11-2 & 50.4 & 2.12 & 15.6 & 8.84 & 14.2 & 0.19 & 3.11 & 2.80 & 0.27 & 0.13 & 0.06 & 0.59 & 98.3 & 0.13 & 0.91 & 65.5 & 82.1 \\
\hline
\end{tabular}


附表 1 (续)

\begin{tabular}{|c|c|c|c|c|c|c|c|c|c|c|c|c|c|c|c|c|c|}
\hline 包裹体 & $\mathrm{SiO}_{2}$ & $\mathrm{TiO}_{2}$ & $\mathrm{Al}_{2} \mathrm{O}_{3}$ & $\mathrm{FeO}_{\mathrm{t}}$ & $\mathrm{CaO}$ & $\mathrm{MnO}$ & $\mathrm{MgO}$ & $\mathrm{Na}_{2} \mathrm{O}$ & $\mathrm{K}_{2} \mathrm{O}$ & $\mathrm{P}_{2} \mathrm{O}_{5}$ & $\mathrm{Cr}_{2} \mathrm{O}_{3}$ & $\mathrm{SO}_{3}$ & Total & $\mathrm{K} / \mathrm{Ti}$ & $\mathrm{CaO} / \mathrm{Al}_{2} \mathrm{O}_{3}$ & $\begin{array}{l}\text { 平衡 } \\
\mathrm{Mg \#}^{\mathrm{a}}\end{array}$ & $\begin{array}{l}\text { 主矿物 } \\
\mathrm{Mg \#}{ }^{\mathrm{b})}\end{array}$ \\
\hline E11-3 & 49.8 & 2.18 & 15.6 & 9.76 & 14.3 & 0.25 & 2.86 & 2.29 & 0.23 & 0.18 & 0.08 & 0.52 & 98.1 & 0.11 & 0.92 & 61.3 & 82.1 \\
\hline E11-4 & 49.4 & 2.08 & 15.1 & 10.4 & 13.7 & 0.20 & 3.92 & 2.55 & 0.21 & 0.13 & 0.12 & 0.63 & 98.4 & 0.10 & 0.91 & 67.1 & 82.2 \\
\hline E11-5 & 49.4 & 2.82 & 14.5 & 10.4 & 13.0 & 0.27 & 4.05 & 2.09 & 0.42 & 0.13 & 0.18 & 0.72 & 98.0 & 0.15 & 0.89 & 67.8 & 82.2 \\
\hline E11-6 & 49.3 & 2.04 & 15.9 & 9.32 & 12.3 & 0.22 & 5.48 & 2.81 & 0.23 & 0.14 & 0.15 & 0.49 & 98.3 & 0.11 & 0.77 & 76.0 & 82.2 \\
\hline E11-7 & 50.0 & 1.71 & 15.1 & 10.1 & 13.5 & 0.15 & 4.12 & 2.53 & 0.15 & 0.16 & 0.12 & 0.47 & 98.1 & 0.09 & 0.89 & 68.8 & 83.4 \\
\hline E11-8 & 49.1 & 1.86 & 15.4 & 10.5 & 13.0 & 0.16 & 5.08 & 2.46 & 0.18 & 0.16 & 0.13 & 0.49 & 98.5 & 0.10 & 0.85 & 72.3 & 83.4 \\
\hline E11-9 & 49.5 & 1.94 & 15.0 & 10.7 & 13.2 & 0.17 & 4.95 & 2.28 & 0.15 & 0.12 & 0.09 & 0.52 & 98.7 & 0.08 & 0.88 & 71.4 & 83.1 \\
\hline E11-10 & 50.4 & 2.02 & 15.4 & 9.51 & 13.8 & 0.16 & 3.34 & 2.68 & 0.12 & 0.24 & 0.15 & 0.54 & 98.3 & 0.06 & 0.90 & 65.5 & 83.1 \\
\hline E11-11 & 50.3 & 1.94 & 14.9 & 10.4 & 13.2 & 0.16 & 4.64 & 2.52 & 0.15 & 0.19 & 0.11 & 0.53 & 99.0 & 0.08 & 0.89 & 70.7 & 83.1 \\
\hline E11-12 & 51.4 & 1.99 & 19.3 & 7.01 & 12.3 & 0.11 & 2.28 & 2.58 & 0.23 & 0.20 & 0.13 & 0.63 & 98.2 & 0.12 & 0.64 & 63.7 & 82.9 \\
\hline E11-13 & 51.8 & 1.98 & 18.1 & 7.44 & 13.0 & 0.14 & 2.05 & 2.79 & 0.18 & 0.13 & 0.11 & 0.55 & 98.3 & 0.09 & 0.72 & 59.8 & 82.9 \\
\hline E11-14 & 50.3 & 1.96 & 14.4 & 10.8 & 13.0 & 0.12 & 4.49 & 2.51 & 0.25 & 0.20 & 0.18 & 0.62 & 98.8 & 0.13 & 0.90 & 69.2 & 82.5 \\
\hline E11-15 & 49.8 & 1.94 & 14.3 & 10.6 & 13.3 & 0.16 & 4.71 & 2.23 & 0.15 & 0.09 & 0.09 & 0.54 & 97.9 & 0.08 & 0.93 & 70.6 & 82.5 \\
\hline
\end{tabular}

a) 平衡 $\mathrm{Mg}$ \#是根据 Ford 等 ${ }^{[34]}$ 计算的与包裹体液相线平衡的橄榄石 $\mathrm{Mg} \# ;$ b) 主矿物 $\mathrm{Mg} \#$ 是通过电子探针在包裹体附近获得的最富 $\mathrm{MgO}$ 的橄榄石 $\mathrm{Mg} \#$ 
附表 2 通过校正橄榄石分异结晶作用获得的初始熔体包裹体组成 $(\%)^{\mathrm{a})}$

\begin{tabular}{|c|c|c|c|c|c|c|c|c|c|c|c|c|c|c|c|c|c|c|c|c|c|c|}
\hline 裏体 & $\mathrm{O}_{2}$ & $\mathrm{T1O}_{2}$ & & $\mathrm{Fe}_{2} \mathrm{O}_{3}$ & $\mathrm{FeO}$ & $\mathrm{FeO}_{\mathrm{t}}$ & $\mathrm{MnO}$ & $\mathrm{MgO}$ & $\mathrm{CaO}$ & $\mathrm{Na}_{2} \mathrm{O}$ & $\mathrm{K}_{2} \mathrm{O}$ & $\mathrm{Cr}_{2} \mathrm{O}_{3}$ & $\mathrm{SO}_{3}$ & $\mathrm{P}_{2} \mathrm{O}_{5}$ & $\mathrm{aaO} / \mathrm{Al}_{2} \mathrm{O}_{3}$ & $\mathrm{Ca}_{8}$ & $\mathrm{Al}_{8}$ & $\mathrm{Ca}_{8} / \mathrm{Al}_{8}$ & $\mathrm{Fe}_{8}$ & $\mathrm{Na}_{8}$ & $F_{\text {melt }}$ & $\mathrm{P} / \mathrm{GPa}$ \\
\hline E42-1 & 48.5 & 1.77 & 13.0 & 1.33 & 10.8 & 12.0 & 0.15 & 10.1 & 12.2 & 1.79 & 0.25 & 0.09 & 0.41 & 0.10 & 0.93 & 11.7 & 11.7 & 1.00 & 12.4 & 2.14 & 23.7 & 0.67 \\
\hline E42-2 & 48.5 & 1.92 & 12.2 & 1.39 & 11.3 & 12.5 & 0.17 & 10.4 & 11.7 & 1.97 & 0.23 & 0.09 & 0.52 & 0.18 & 0.96 & 11.3 & 10.8 & 1.05 & 12.8 & 2.37 & 23.2 & .68 \\
\hline E42-3 & 48.7 & 1.91 & 13.2 & 1.28 & 10.4 & 11.6 & 0.19 & 9.54 & 2.1 & 2.19 & 0.22 & 0.14 & 0.44 & 0.13 & 0.91 & 11.6 & 12.1 & 0.96 & 11.9 & 2.45 & 21.4 & 0.54 \\
\hline E42-4 & 49.2 & 1.87 & 13.6 & 1.25 & 10.1 & 11.2 & 0.14 & 9.31 & 12.0 & 2.10 & 0.21 & 0.11 & 0.45 & 0.13 & 0.88 & 11.5 & 12.6 & 0.92 & 11.5 & 2.32 & 21.4 & 0.51 \\
\hline E20-1 & 48.5 & 1.28 & 14.9 & 1.06 & 8.62 & 9.57 & 0.13 & 10.8 & 12.7 & 1.75 & 0.14 & 0.20 & 0.48 & 0.01 & 0.85 & 12.5 & 13.4 & 0.93 & 9.92 & 2.23 & 22.1 & 0.90 \\
\hline E20-2 & 48.2 & 1.29 & 15.6 & 1.01 & 8.22 & 9.13 & 0.15 & 10.2 & 12.8 & 2.04 & 0.11 & 0.13 & 0.35 & 0.21 & .82 & 12.4 & 14.2 & 0.87 & 9.78 & 2.42 & 20.2 & 0.88 \\
\hline E20-3 & 49.0 & 1.52 & 16.1 & 1.02 & 8.28 & 9.20 & 0.14 & 8.92 & 12.3 & 2.20 & 0.20 & 0.19 & 0.39 & 0.15 & 0.77 & 11.9 & 15.3 & 0.78 & 9.37 & 2.36 & 19.1 & 0.68 \\
\hline E20-4 & 48.7 & 1.46 & 15.2 & 1.11 & 9.02 & 10.0 & 0.11 & 9.7 & 2.0 & 2.18 & 0.18 & 0.15 & 0.36 & 0.13 & 0.79 & 11.5 & 14.0 & 0.82 & 10.6 & 2.47 & 19.1 & 0.84 \\
\hline E20-5 & 49.4 & 1.44 & 16.2 & 1.01 & 8.18 & 9.08 & 0.09 & 8.76 & 11.8 & 2.57 & 0.21 & 0.21 & 0.37 & 0.15 & 0.73 & .5 & 15.6 & 0.74 & 9.12 & 2.70 & 16.7 & 0.70 \\
\hline E20-6 & 49.3 & 1.56 & 15.2 & 1.08 & 8.77 & 9.74 & 0.09 & 9.37 & 11.9 & 2.25 & 0.26 & 0.15 & 0.34 & 0.14 & 0. & 11.4 & 14.1 & 0.81 & 10.2 & 2.48 & 18.9 & 0.71 \\
\hline E15-1 & 47.8 & 1.30 & 15.6 & 1.08 & 8.78 & 9.75 & 0.20 & 10.0 & 12.9 & 2.00 & 0.15 & 0.12 & 0.29 & 0.05 & 0.82 & 12.4 & 14.3 & 0.87 & 10.4 & 2.34 & 20.6 & 0.92 \\
\hline E15-2 & 47.7 & 1.33 & 15.1 & 1.13 & 9.13 & 10.1 & 0.05 & 10.4 & 12.8 & 1.96 & 0.14 & 0.14 & 0.30 & 0.09 & 85 & 12.5 & 13.7 & 0.91 & 10.6 & 2.38 & 21.0 & 0.96 \\
\hline E15-3 & 49.2 & 50 & 17.3 & 0.80 & 6.48 & 7.21 & 0.06 & 7. & 14.2 & 2.61 & 0.08 & 0.14 & 0.48 & 0.19 & 0. & 1.5 & 17.7 & 0.82 & 5.82 & 2.53 & 18.8 & 17 \\
\hline E15-4 & 47.8 & 1.42 & 15.3 & 1.08 & 8.74 & 9.72 & 0.26 & 9.95 & 12.8 & 2.12 & 0.16 & 0.19 & 0.37 & 0.18 & 0.84 & 12.3 & 14.0 & 0.88 & 10.3 & 2.45 & 20.2 & 0.85 \\
\hline E15-5 & 47.0 & 1.35 & 14.0 & 1.20 & 9.73 & 10.8 & 0.13 & 11.4 & 13.1 & 1.67 & 0.12 & 0.35 & 0.39 & 0.00 & 0.93 & 13.4 & 12.8 & 1.05 & 10.4 & 2.27 & 23.8 & 1.07 \\
\hline E15-6 & 47.6 & 1.33 & 14.1 & 1.17 & 9.45 & 10 & 0.18 & 11.1 & 13.0 & 86 & 0.11 & 0.19 & 0.32 & 0.00 & 0.92 & 13.0 & 12.7 & 1.02 & 10.5 & 2.40 & 22.7 & 0.96 \\
\hline E15-7 & 47.3 & 1.32 & 13.9 & 1.19 & 9.64 & 10.7 & 0.23 & 11.4 & 12.9 & 1.70 & 0.05 & 0.23 & 0.34 & 0.13 & 0.93 & 13.3 & 12.6 & 1.05 & 10.3 & 2.30 & 23.6 & 1.02 \\
\hline E15-8 & 48.1 & 1.50 & 15.5 & 1.02 & 8.25 & 9.17 & 0.18 & 9.56 & 13.2 & 2.26 & 0.14 & 0.08 & 0.57 & 0.14 & 0. & 12.7 & 14.4 & 0.89 & 9.75 & 2.53 & 19.9 & 0.66 \\
\hline E15-9 & 47.8 & 1.39 & 15.7 & 0.98 & 7.92 & 8.80 & 0.13 & 9.03 & 14.1 & 2.47 & 0.17 & 0.18 & 0.47 & 0.10 & 0.89 & 13.6 & 14.9 & 0.92 & 9.10 & 2.64 & 19.7 & 0.47 \\
\hline E15-10 & 47.8 & 1.37 & 14.9 & 1.09 & 8.84 & 9.82 & 0.19 & 10.4 & 12.8 & 2.13 & 0.10 & 0.20 & 0.31 & 0.11 & 0.86 & 12.5 & 13.5 & 0.92 & 10.3 & 2.54 & 20.4 & 0.89 \\
\hline E15-11 & 47.7 & 1.34 & 15.4 & 1.06 & 8.57 & 9.52 & 0.20 & 10.0 & 13.2 & 2.02 & 0.17 & 0.16 & 0.35 & 0.07 & 0.86 & 12.8 & 14.1 & 0.91 & 10.1 & 2.37 & 21.1 & 0.82 \\
\hline E13-1 & 47.5 & 1.38 & 14.0 & 1.19 & 9.60 & 10.7 & 0.16 & 11.1 & 12.6 & 2.04 & 0.18 & 0.18 & 0.36 & 0.18 & 0.90 & 12.6 & 12.6 & 1.00 & 10.7 & 2.57 & 21.4 & 1.00 \\
\hline E13-2 & 47.7 & 1.36 & 14.4 & 1.16 & 9.37 & 10.4 & 0.17 & 10.9 & 12.6 & 1.86 & 0.18 & 0.14 & 0.28 & 0.12 & 0.88 & 12.6 & 13.0 & 0.97 & 10.6 & 2.36 & 22.0 & 0.98 \\
\hline E13-3 & 47.0 & 1.54 & 11.8 & 1.37 & 11.1 & 12.3 & 0.15 & 12.9 & 12.3 & 1.16 & 0.37 & 0.17 & 0.42 & 0.08 & 1.05 & 14.9 & 12.3 & 1.21 & 8.80 & 2.15 & 26.9 & 1.23 \\
\hline E13-4 & 48.1 & 1.52 & 15.9 & 1.00 & 8.08 & 8.98 & 0.17 & 9.32 & 13.5 & 2.07 & 0.16 & 0.16 & 0.47 & 0.09 & 0.85 & 13.0 & 14.9 & 0.87 & 9.47 & 2.30 & 20.8 & 0.64 \\
\hline E13-5 & 47.7 & 1.39 & 15.0 & 1.10 & 8.94 & 9.93 & 0.17 & 10.3 & 12.8 & 2.07 & 0.20 & 0.18 & 0.43 & 0.13 & 0.86 & 12.5 & 13.6 & 0.92 & 10.5 & 2.46 & 20.7 & 0.89 \\
\hline E13-6 & 47.8 & 1.39 & 14.6 & 1.13 & 9.18 & 10.2 & 0.16 & 10.7 & 12.7 & 1.88 & 0.19 & 0.14 & 0.37 & 0.08 & 0.87 & 12.5 & 13.2 & 0.95 & 10.6 & 2.34 & 21.8 & 0.94 \\
\hline E13-7 & 48.0 & 1.39 & 15.3 & 1.07 & 8.69 & 9.66 & 0.21 & 10.1 & 13.0 & 1.91 & 0.21 & 0.13 & 0.44 & 0.00 & 0.85 & 12.6 & 13.9 & 0.90 & 10.3 & 2.26 & 21.5 & 0.82 \\
\hline E13-8 & 48.2 & 1.49 & 16.0 & 1.00 & 8.08 & 8.98 & 0.14 & 9.2 & 12.6 & 2.59 & 0.23 & 0.20 & 0.41 & 0.19 & 0.79 & 12.2 & 15.1 & 0.81 & 9.42 & 2.81 & 17.2 & 0.75 \\
\hline E13-9 & 48.5 & 1.24 & 16.9 & 1.01 & 8.21 & 9.12 & 0.17 & 10.0 & 11.2 & 2.24 & 0.19 & 0.18 & 0.41 & 0.09 & 0.66 & 10.7 & 15.6 & 0.69 & 9.78 & 2.58 & 16.5 & 1.26 \\
\hline E13-10 & 49.0 & 1.31 & 16.2 & 0.98 & 7.90 & 8.78 & 0.14 & & 12.3 & 2.08 & 0.19 & 0.13 & 0.38 & 0.11 & 0.76 & 11.8 & 15.1 & 0.79 & 9.42 & 2.36 & 19.2 & 0.84 \\
\hline E13-11 & 50.0 & 1.28 & 17.8 & 0.89 & 7.18 & 7.97 & 0.13 & 8.73 & 10.7 & 2.82 & 0.21 & 0.11 & 0.45 & 0.09 & 0.60 & 10.4 & 17.2 & 0.60 & 8.09 & 2.94 & 13.3 & 0.99 \\
\hline E13-12 & 48.6 & 1.30 & 15.9 & 1.03 & 8.35 & 9.28 & 0.15 & 10.2 & 11.9 & 2.24 & 0.20 & 0.16 & 0.37 & 0.07 & 0.75 & 11.5 & 14.5 & 0.79 & 9.91 & 2.62 & 18.0 & 1.02 \\
\hline E13-13 & 7.9 & 1.26 & 15.2 & 1.12 & 9.06 & 10.1 & 0.11 & 10.7 & 12.4 & 1.89 & 0.13 & 0.12 & 0.42 & 0.14 & 0.82 & 12.2 & 13.8 & 0.89 & 10.4 & 2.35 & 20.8 & 1.06 \\
\hline E13-14 & 7.9 & 1.23 & 15.5 & 1.08 & 8.78 & 9.75 & 0.15 & 10.6 & 12.3 & 2.00 & 0.17 & 0.13 & 0.41 & 0.14 & 0.80 & 12.1 & 14.0 & 0.86 & 10.2 & 2.44 & 19.9 & 1.08 \\
\hline E13-15 & 7.7 & 1.32 & 14.7 & 1.16 & 9.37 & 10.4 & 0.07 & 11.2 & 11.9 & 2.16 & 0.17 & 0.19 & 0.18 & 0.09 & 0.81 & 12.0 & 13.4 & 0.90 & 10.3 & 2.73 & 19.0 & 1.23 \\
\hline E13-16 & 7.6 & 1.28 & 14.9 & 1.15 & 9.28 & 10.3 & 0.14 & 11.1 & 12.2 & 1.96 & 0.15 & 0.07 & 0.44 & 0.16 & 0.8 & 12.3 & 13.6 & 0.91 & 10.2 & 2.51 & 20.3 & 1.19 \\
\hline E13-17 & 7.3 & 1.33 & 13.7 & 1.23 & 9.92 & 11.0 & 0.18 & 11.7 & 12.1 & 2.18 & 0.18 & 0.12 & 0.41 & 0.10 & 0.88 & 12.7 & 12.6 & 1.01 & 10.2 & 2.85 & 20.1 & 1.19 \\
\hline E13-18 & 8.2 & 1.35 & 15.8 & 1.04 & 8.40 & 9.33 & 0.15 & 10.1 & 12.4 & 2.20 & 0.15 & 0.11 & 0.34 & 0.08 & 0.78 & 11.9 & 14.5 & 0.82 & 9.97 & 2.55 & 18.8 & 0.97 \\
\hline E13-19 & 7.8 & 1.13 & 14.8 & 1.16 & 9.3 & 10.4 & 0.12 & 11.3 & 12.1 & 1.90 & 0.13 & 13 & 0.34 & 0.13 & 0.8 & 12.4 & 3.5 & 0.92 & 10.1 & 2.49 & 20.5 & 1.23 \\
\hline E13-20 & 8.0 & 1.19 & 15.5 & 1.09 & 8.81 & 9.79 & 0.14 & 10.7 & 12.2 & 2.14 & 0.13 & 0.12 & 0.53 & 0.11 & 0.79 & 12.0 & 14.0 & 0.85 & 10.2 & 2.60 & 19.0 & 1.11 \\
\hline E13-21 & 48.3 & 1.30 & 15.9 & 1.02 & 8.25 & 9.17 & 0.17 & 10.0 & 12.4 & 2.18 & 0.14 & 0.08 & 0.47 & 0.16 & 0.78 & 12.0 & 14.6 & 0.82 & 9.83 & 2.53 & 18.9 & 0.94 \\
\hline E13-22 & 8.8 & 1.20 & 16.2 & 0.98 & 7.97 & 8.85 & 0.16 & 9.64 & 12.2 & 2.36 & 0. & 0.14 & 0.34 & 0.17 & 0.75 & 11.7 & 15.0 & 0.78 & 9.49 & 2.64 & 17.7 & 0.88 \\
\hline E13-23 & 7.8 & 1.26 & 15.4 & 1.11 & 8.98 & 10.0 & 0.15 & 10.6 & 12.5 & 1.87 & 0.13 & 0.20 & 0.35 & 0.11 & 0.81 & 12.2 & 14.0 & 0.87 & 10.4 & 2.31 & 20.8 & 1.09 \\
\hline E13-24 & 48.4 & 1.29 & 15.8 & 1.04 & 8.43 & 9.37 & 0.07 & 10.1 & 12.3 & 2.05 & 0.15 & 0.12 & 0.51 & 0.14 & 0.78 & 11.9 & 14.5 & 0.82 & 10.0 & 2.41 & 19.5 & 0.96 \\
\hline E13-25 & 49.0 & 1.33 & 16.5 & 0.97 & 7.89 & 8.76 & 0.15 & 9.50 & 11.9 & 2.38 & 0.16 & 0.13 & 0.45 & 0.20 & 0.72 & 11.4 & 15.3 & 0.74 & 9.36 & 2.63 & 17.1 & 0.91 \\
\hline E13-26 & 48.9 & 1.26 & 17.2 & 0.94 & 7.63 & 8.48 & 0.11 & 8.96 & 11.7 & 2.77 & 0.26 & 0.10 & 0.42 & 0.11 & 0.68 & 11.3 & 16.5 & 0.69 & 8.76 & 2.94 & 14.7 & 0.92 \\
\hline E13-27 & 47.8 & 1.14 & 15.4 & 1.11 & 8.98 & 10.0 & 0.12 & 11.1 & 11.9 & 2.08 & 0.15 & 0.10 & 0.29 & 0.14 & 0.77 & 12.0 & 14.0 & 0.86 & 9.98 & 2.62 & 18.9 & 1.27 \\
\hline 3-28 & 48.1 & 1.16 & 15.7 & 1.06 & 8.58 & 9.53 & 0.13 & 10.6 & 12.2 & 2.05 & 0.18 & 0.16 & 0.33 & 0.15 & 0.78 & 11. & 14.2 & 0.84 & 9.96 & 2.51 & 19.3 & 1.13 \\
\hline E13-29 & 48.1 & 1.14 & 15.8 & 1.01 & 8.21 & 9.12 & 0.08 & 9.87 & 13.3 & 1.96 & 0.20 & 0.16 & 0.31 & 0.14 & 0.84 & 12.8 & 14.5 & 0.88 & 9.78 & 2.27 & 21.2 & 0.79 \\
\hline E11-1 & 48.4 & 1.78 & 13.2 & 1.40 & 11.3 & 12.6 & 0.22 & 9.13 & 12.1 & 2.11 & 0.19 & 0.17 & 0.48 & 0.07 & 0.91 & 11.6 & 12.3 & 0.94 & 12.6 & 2.30 & 21.9 & 0.55 \\
\hline E11-2 & 49.3 & 1.82 & 13.4 & 1.30 & 10.5 & 11.7 & 0.20 & 8.46 & 12.2 & 2.40 & 0.23 & 0.06 & 0.51 & 0.11 & 0.91 & 12.0 & 13.0 & 0.92 & 11.1 & 2.48 & 20.7 & 0.27 \\
\hline E11-3 & 48.1 & 1.76 & 12.6 & 1.49 & 12.1 & 13.4 & 0.25 & 9.85 & 11.6 & 1.85 & 0.19 & 0.07 & 0.42 & 0.15 & 0.92 & 11.1 & 11.3 & 0.98 & 13.7 & 2.16 & 23.3 & 0.75 \\
\hline
\end{tabular}


附表 2(续)

\begin{tabular}{|c|c|c|c|c|c|c|c|c|c|c|c|c|c|c|c|c|c|c|c|c|c|c|}
\hline 裹体 & $\mathrm{SiO}_{2}$ & $\mathrm{TiO}_{2}$ & $\mathrm{Al}_{2}$ & & $\mathrm{eO}$ & $\mathrm{FeO}_{\mathrm{t}}$ & $\mathrm{MnO}$ & $\mathrm{MgO}$ & $\mathrm{CaO}$ & $\mathrm{Na}_{2} \mathrm{O}$ & $\mathrm{K}_{2} \mathrm{O}$ & $\mathrm{Cr}_{2} \mathrm{O}_{3}$ & $\mathrm{SO}_{2}$ & $\mathrm{P}_{2} \mathrm{O}_{5}$ & $\mathrm{O}_{1} \mathrm{H}_{2} \mathrm{O}_{3}$ & $\mathrm{Ca}_{8}$ & $\mathrm{Al}_{8}$ & $\mathrm{Ca}_{8} / \mathrm{Al}_{8}$ & $\mathrm{Fe}_{8}$ & $\mathrm{Na}_{8}$ & $\mathrm{~F}_{\text {melt }}$ & $\mathrm{P} / \mathrm{GPa}$ \\
\hline E11-4 & 48.2 & 1.75 & 12.7 & 1.47 & 11.9 & 13.2 & 0.21 & 9.65 & 11.6 & 2.15 & 0.18 & 0.11 & 0.53 & 0.11 & 0.91 & 11.1 & 11.5 & 0.96 & 13.4 & 2.43 & 21.6 & 0.7 \\
\hline E11-5 & 48.6 & 2.42 & 12.5 & 1.43 & 11.6 & 12.9 & 0.27 & 9.54 & 11.2 & 1.80 & 0.36 & 0.16 & 0.62 & 0.11 & 0.90 & 10.7 & 11.3 & 0.95 & 13.1 & 2.06 & 23.3 & 0.6 \\
\hline E11-6 & 49.7 & 1.98 & 15.4 & 1.12 & 9.04 & 10.0 & 0.23 & 7.31 & 11.9 & 2.73 & 0.22 & 0.15 & 0.48 & 0.14 & 0.77 & 12.4 & 16.1 & 0.77 & 7.93 & 2.60 & 17.8 & 0.25 \\
\hline E11-7 & 49.0 & 1.46 & 12.8 & 1.38 & 11.2 & 12.4 & 0.16 & 9.93 & 11.5 & 2.15 & 0.13 & 0.11 & 0.40 & 0.14 & 0.90 & 11.1 & 11.5 & 0.96 & 12.8 & 2.48 & 21.3 & 0.69 \\
\hline E11-8 & 48.7 & 1.67 & 13.8 & 1.32 & 10.7 & 11.8 & 0.17 & 9.39 & 11.7 & 2.21 & 0.16 & 0.12 & 0.44 & 0.14 & 0.85 & 11.2 & 12.7 & 0.88 & 12.1 & 2.44 & 20.2 & 0.69 \\
\hline E11-9 & 48.5 & 1.68 & 13.0 & 1.42 & 11.5 & 12.7 & 0.18 & 10.0 & 11.5 & 1.97 & 0.13 & 0.09 & 0.45 & 0.10 & 0.88 & 11.0 & 11.6 & 0.95 & 13.0 & 2.31 & 22.0 & 0.78 \\
\hline E11-10 & 49.1 & 1.69 & 12.9 & 1.36 & 11.0 & 12.2 & 0.17 & 9.55 & 11.6 & 2.24 & 0.10 & 0.13 & 0.45 & 0.20 & 0.90 & 11.1 & 11.7 & 0.95 & 12.5 & 2.51 & 20.9 & 0.57 \\
\hline E11-11 & 49.0 & 1.66 & 12.8 & 1.39 & 11.3 & 12.5 & 0.17 & 9.83 & 11.4 & 2.16 & 0.13 & 0.10 & 0.45 & 0.16 & 0.89 & 10.9 & 11.5 & 0.95 & 12.8 & 2.47 & 21.1 & 0.68 \\
\hline E11-12 & 50.7 & 1.77 & 17.1 & 0.99 & 8.00 & 8.9 & 0.13 & 7.21 & 11.0 & 2.59 & 0.20 & 0.12 & 0.56 & 0.18 & 0.64 & 11.5 & 17.9 & 0.65 & 6.73 & 2.44 & 16.6 & 0.52 \\
\hline E11-13 & 50.6 & 1.71 & 15.6 & 1.11 & 8.98 & 10.0 & 0.15 & 7.80 & 11.3 & 2.40 & 0.16 & 0.10 & 0.47 & 0.11 & 0.72 & 11.4 & 15.8 & 0.72 & 8.67 & 2.37 & 18.2 & 0.45 \\
\hline E11-14 & 48.8 & 1.65 & 12.1 & 1.49 & 12.0 & 13.4 & 0.14 & 10.1 & 11.0 & 2.11 & 0.21 & 0.16 & 0.52 & 0.17 & 0.91 & 10.6 & 10.7 & 0.98 & 13.6 & 2.47 & 21.7 & 0.75 \\
\hline E11-15 & 49.0 & 1.67 & 12.3 & 1.46 & 11.8 & 13.1 & 0.18 & 9.93 & 11.5 & 1.92 & 0.13 & 0.08 & 0.46 & 0.08 & 0.93 & 11.0 & 11.0 & 1.00 & 13.4 & 2.25 & 23.2 & 0.65 \\
\hline
\end{tabular}

a) $\mathrm{Ca}_{8}, \mathrm{Al}_{8}, \mathrm{Fe}_{8}$ 和 $\mathrm{Na}_{8}$ 分别为相对 $\mathrm{MgO}=8.0 \mathrm{wt} \%$ 校正地壳内低压分异结晶作用后的 $\mathrm{CaO}, \mathrm{Al}_{2} \mathrm{O}_{3}, \mathrm{FeO}_{\mathrm{t}}$ 和 $\mathrm{Na}_{2} \mathrm{O}$ 含量; $\mathrm{F}_{\text {melt }}$ 为根据 $\mathrm{Niu}$ 等人 计算的二辉橄榄岩地幔源区部分熔融程度; P 为根据 Yang 等人 ${ }^{[60]}$ 计算的岩浆结晶出单斜辉石时的压力 